\title{
Towards achieving efficient MAC protocols for WBAN-enabled loT technology: a review
}

\author{
Damilola D. Olatinwo ${ }^{1}$, Adnan M. Abu-Mahfouz ${ }^{1,2^{*}}$ and Gerhard P. Hancke ${ }^{1,3}$
}

\section{${ }^{*}$ Correspondence:}

a.abumahfouz@ieee.org

${ }^{1}$ Department of Electrical,

Electronic and Computer

Engineering, University

of Pretoria, Pretoria 0001

South Africa

Full list of author information

is available at the end of the

article

\begin{abstract}
Internet of things (loT) is a concept that is currently gaining a lot of popularity as a result of its potential to be incorporated into many heterogeneous systems. Because of its diversity, integrating loT is conceivable in almost all fields, including the healthcare sector. For instance, a promising technology in the healthcare sector known as wireless body area network (WBAN) could be integrated with the loT to enhance its productivity. However, in order to guarantee the optimization of the operation of the healthcare applications facilitated by the WBAN-enabled loT technology, there must be enough support from all the different protocol stack layers so as to satisfy the critical qualityof-service (QoS) requirements of the WBAN systems. Consequently, the medium access control (MAC) protocol has recently been gaining lots of attention in the area of WBANs due to its ability to manage and coordinate when a shared communication channel can be accessed. For the purpose of achieving efficient MAC protocols for WBAN-enabled loT technology, this paper investigates some key MAC protocols that could be exploited in WBANs based on their characteristics, service specifications, technical issues such as energy wastage issues, and possible technical solutions were provided to enhance energy efficiency, channel utilization, data transmission rate, and dealy rate. Also, these MAC protocols were grouped and compared based on shortand long-range communication standards. Following this, future directions and open research issues are pointed out.
\end{abstract}

Keywords: IoT, 5G technology, Energy efficiency, Communication standards, WBAN, MAC protocols

\section{Introduction}

To improve the quality-of-life, a wireless body area network (WBAN) has been specifically designed for healthcare monitoring [1]. The agenda of this type of wireless network is to provide health related services that targets the general well-being of humans by regularly monitoring critical vital signs, including the heartbeat, respiration, temperature, and blood pressure, collecting specialized signals, like the electromyography (EMG), electroencephalogram (EEG), and electrocardiogram (ECG), and reporting the obtained measurements remotely to dedicated physicians and hospitals via communication technologies, reducing hospital visits in a cost-effective manner, and minimizing the rate of hospitalization. As an internet communication paradigm, the internet of things (IoT)

(c) The Author(s) 2021. Open Access This article is licensed under a Creative Commons Attribution 4.0 International License, which permits use, sharing, adaptation, distribution and reproduction in any medium or format, as long as you give appropriate credit to the original author(s) and the source, provide a link to the Creative Commons licence, and indicate if changes were made. The images or other third party material in this article are included in the article's Creative Commons licence, unless indicated otherwise in a credit line to the material. If material is not included in the article's Creative Commons licence and your intended use is not permitted by statutory regulation or exceeds the permitted use, you will need to obtain permission directly from the copyright holder. To view a copy of this licence, visit http:// creativecommons.org/licenses/by/4.0/. 
technology has gained a lot of attention recently in the academia sphere as well as industry because of its ability to be applied to various wireless systems and seamlessly connect various types of devices, such as actuators, sensors, medical devices, and vehicles together over the internet to achieve the critical tasks of different wireless networks, like WBAN applications, pervasively. IoT technologies can be fused with WBANs to form a WBAN-enabled IoT technology for enhanced functionalities and new use cases [1]. Such systems are not only suitable for healthcare monitoring, but also have capabilities to autonomously carry out process control and decision-making functions with or without any human intervention [2]. These systems consist of intelligent, miniature size, and low-power IoT biosensor devices which are positioned inside, around or worn on the human body to monitor, diagnose, and treat patients that suffers from chronic diseases, including cancer, diabetes, obesity, myocardial infraction, stroke, and other forms of diseases, in a seamless manner, as well as communicate sensory health data to healthcare providers, including designated hospitals [1-3], through access point (AP) or base station (BS) nodes in the body area.

Because of the aforementioned unique properties, the WBAN-enabled IoT technology must be well supported in terms of energy efficiency and/or long lifetime requirement, reasonable latency, efficient channel bandwidth utilization, long communication coverage, low power utilization, pervasiveness, low-cost deployment, and sufficient throughput to efficiently drive these systems as a result of inherent resource scarcity issues related them. Given that the biosensor devices in these systems have limited battery power resources since they mostly use batteries [4], and the energy expended during health data communications is always huge and expensive compared to other activities, then, it will be reasonable and advantageous to reduce the power consumption due to data communications through pragmatic means, including medium access control (MAC) mechanisms and/or power control mechanisms. For insight into data communications in WBAN systems, an illustration is provided in Fig. 1.

Because of the MAC layer energy consumption and/or energy wastage associated issues when biosensor devices try to access the communication channel, this study is focused on addressing the concerns of the MAC layer that are associated with the WBAN systems, and seeking the design of good MAC protocols that are characterized

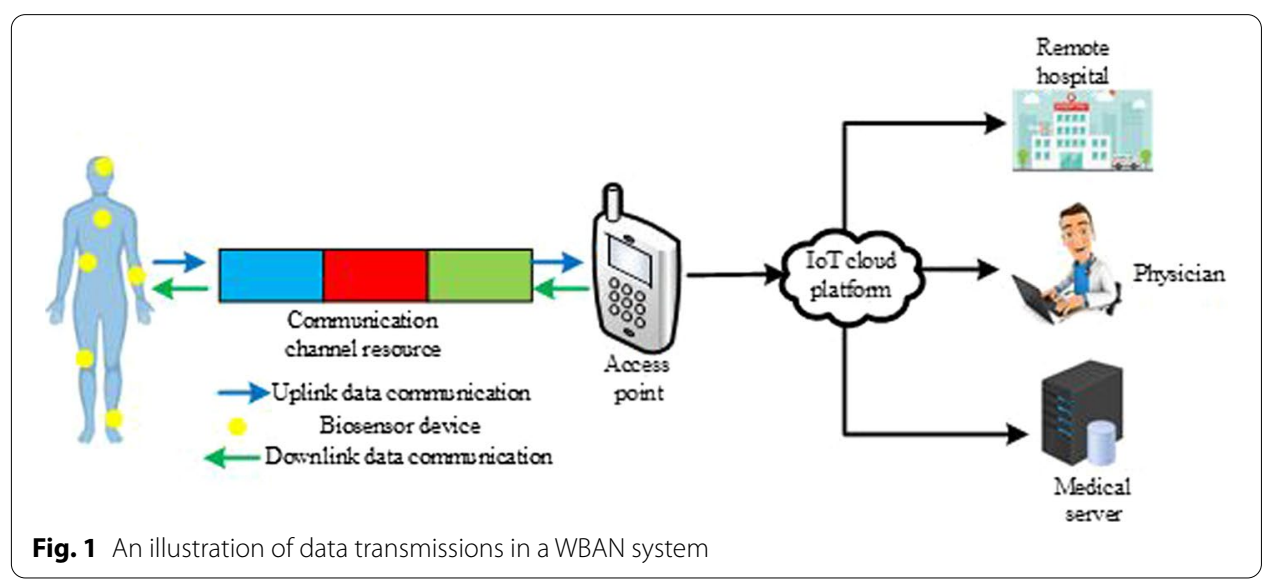


by efficiency, low energy consumption, reliability, time sensitivity, low latency, reasonable data transfer rate, as well as low hardware implementation cost [4].

Currently, the investigation and design of efficient MAC protocols for WBAN systems are active research areas in the healthcare research sphere [5, 6]. The design of efficient MAC protocols for WBANs is a promising adventure that would be highly helpful to optimally control how the biosensor devices access the communication channel, and improve the allocation of the WBAN system scarce network resources, including time slots, power, spectrum and/or channel. Hence, this study reviews some MAC protocols that could be exploited in WBANs and those designed specifically for the WBAN systems. The main contributions of this research work are highlighted as follows:

- General overview of wireless MAC protocols that can be exploited in the WBANs.

- Exploration of MAC protocols that are specific to WBANs.

- Identification of suitable MAC protocols by exploring their characteristics, service specifications, technical issues such as energy wastage issues, and possible solutions.

- Identification of relevant communication technologies in line with the WBAN IoTrelated MAC protocols.

- Investigation and comparison of different MAC protocols based on short- and longrange standards in relation to energy efficiency, health data transmission rate, radio access techniques, and delay rate.

- Provision of insights into relevant MAC protocols, including their energy consumption, issues, and energy saving mechanisms.

- Presentation of recommendations and future directions.

To authors' best knowledge, this research study is the first to investigate and compare WBAN system MAC protocols based on short- and long-range coverage area with focus on energy consumption issues, channel access techniques, transmission efficiency, and latency. Additionally, this study was able to highlight the protocols used by different communication standards, their energy consumption, energy saving mechanisms, and related issues. The remaining parts of this study are structured as follows. Section 2 presents a review of some existing survey papers for comparison purposes. MAC protocol requirements for WBAN-enabled IoT technology are presented in Sect. 3. Section 4 discuss the general overview of some MAC protocols in communication systems and WBAN-enabled IoT technology. Short- and long-range MAC protocols are presented in Sect. 5. Open research problems as well as future directions are presented in Sect. 6, while Sect. 7 concludes this study.

\section{Related survey paper}

Due to the scarceness of energy resources in wireless communication systems, such as the WBAN systems, research interest has recently been drawn toward exploiting efficient channel access protocols to address the energy consumption problem without compromising the application stringent quality of service (QoS) requirements, like reliability, delay-rate, and throughput. As an example, the authors of [7] focused on identifying the research challenges associated with the design of hybrid MAC protocols that are based on the IEEE 802.15.6 standard which falls under the category of short-range 
communication technologies for exploitation in WBANs. Unlike this work, we focused on MAC protocols for both short- and long-range communication standards for the WBAN systems. Another example is [8], authors presented a research work on WBAN for e-health with a major focus on the MAC protocols challenges and a context-aware design was introduced to achieve an efficient and a reliable network. Their discussions were based on the IEEE wireless standards that include the IEEE 802.15.4 and IEEE 802.15.6. In contrast to [8], we presented a study that focuses on efficient MAC protocols that can be exploited in the WBAN systems by putting into consideration their service requirements and technical challenges related to energy efficiency. Additionally, we investigate as well as compare different MAC protocols based on short- and long-range communication standards and related issues, including energy wastage like collisions, idle listening, as well as radio access techniques, energy saving mechanisms, health data transmission rate, delay rate, and transmission efficiency.

In [9], authors presented a survey on the existing MAC protocols used in WBAN with focus on the requirements, sources of energy wastage, and classification. Also, they briefly discussed the IEEE 802.15.4 and IEEE 802.15.4 wireless standards MAC protocols. Different from [9], we investigated the use of 5G IoT radio standards and technologies, such as the eMTC and NB-IoT MAC protocols in WBAN systems for energy efficiency. Furthermore, in our study, we grouped different MAC protocols based on short and long range so as to know the optimal MAC protocol(s) to employ when considering the design of a WBAN-enabled IoT healthcare system for different use cases by comparing the MAC protocols that falls into each group and also outlining their weaknesses and strengths.

A survey was carried out on MAC protocols in [10], and the authors considered some sources of energy wastage, design requirements, and the advantages and disadvantages of the MAC protocols. Since the work in [10] did not consider the grouping of MAC protocols according to their communication coverage, this limitation consequently creates a context for a new survey, so we presented a study that is different from [10] which classifies MAC protocols into short- and long-range coverage, and also the exploitation of $5 \mathrm{G}$ IoT radio standards and technologies MAC protocols which can be used to achieve energy efficiency in a WBAN system was introduced. The authors of [11] presented a study on the existing MAC protocols designed for WBANs with focus on their salient characteristics and sources of energy wastage. The only wireless standard considered here was IEEE 802.15.4. The authors of [12] reviewed MAC protocols for WBANs, and they considered the design objectives of the IEEE 802.15.6 and IEEE 802.15.4 based MAC superframe structures. Also, the design goals of various multiple access (MA) schemes and their challenges were discussed. In [13], four different MAC protocols that include Medical MAC (MedMAC), energy efficient MAC, BodyMAC, and low duty cycle MAC were investigated. Sources of energy wastage and some important MAC protocol requirements were also discussed. Authors of [14] investigated some energyaware MAC protocols with their optimization methods, and they also analyzed and compared the path loss for WBASN communications. Different from what were done in [11-14], we presented a study that focuses on achieving efficient MAC protocols that can be exploited in WBAN systems by putting into consideration their service requirements and technical challenges related to energy wastage. In addition, we investigated 
and compared different MAC protocols based on short- and long-range standards with focus on radio access techniques and issues related to energy wastage. In [15], a survey based on the MAC protocols used in wireless sensor networks (WSNs) was presented, and the survey focused on the two major categories of the generic MAC protocols that include the contention and scheduled based MAC protocols. The survey investigated some short-range wireless MAC protocol standards that could be employed in WSNs, such as the IEEE 802.11, IEEE 802.15.4, and Bluetooth. Since the survey investigated in [15] is limited to the short-range wireless standards, we therefore explored the investigation of various MAC protocols applicable to long-range communication networking, including the new $5 \mathrm{G}$ IoT radio standards.

The focus of the survey in [16] was on both short- and long-range MAC protocols for IoT, but not for WBAN systems. Also, the considered MAC protocols were classified and compared based on energy consumption, transmission power, advantages, and disadvantages. To complement [16], this new study focused on an IoT-enabled WBAN healthcare system, and we also introduced the exploration of $5 \mathrm{G}$ IoT radio standards and technologies MAC protocols in IoT-enabled WBAN healthcare systems to improve energy efficiency, latency, and throughput network performances. Also, efficient WBAN MAC protocol service requirements and technical challenges are discussed in this study. Furthermore, different from [16], we present a general overview of MAC protocols used in wireless communication systems and MAC protocols that are specific to WBANs.

For the purpose of comparison, the surveyed related papers and this study are summarized and compared in Table 1.

\section{MAC protocol requirements for WBAN-enabled loT technology}

The analysis of MAC protocol requirements is an inseparable part of the WBAN-enabled IoT technology, and hence, this necessitates a quick review of WBAN-enabled IoT technology MAC protocol requirements. Consequently, this section presents some of the key design requirements to be considered in the development and deployment of a MAC protocol for WBAN-enabled IoT healthcare systems. Examples include energy efficiency, latency, reliability, fairness, low complexity, and throughput performance metrics. These parameters are discussed in the next subsections.

Table 1 Comparison of related survey work on short- and long-range MAC protocols for WBAN systems

\begin{tabular}{llll}
\hline $\begin{array}{l}\text { Survey on MAC } \\
\text { protocols (Reference) }\end{array}$ & $\begin{array}{l}\text { Short-range MAC } \\
\text { protocols }\end{array}$ & $\begin{array}{l}\text { Concept of long-range MAC } \\
\text { protocols for WBAN systems }\end{array}$ & $\begin{array}{l}\text { Concept of 5G-enabled MAC } \\
\text { protocols in WBAN systems }\end{array}$ \\
\hline$[7]$ & $\checkmark$ & $\times$ & $\times$ \\
{$[8]$} & $\checkmark$ & $\times$ & $\times$ \\
{$[9]$} & $\checkmark$ & $\times$ & $\times$ \\
{$[10]$} & $\checkmark$ & $\times$ & $\times$ \\
{$[11]$} & $\checkmark$ & $\times$ & $\times$ \\
{$[12]$} & $\checkmark$ & $\times$ & $\times$ \\
{$[13]$} & $\checkmark$ & $\times$ & $\times$ \\
{$[14]$} & $\checkmark$ & $\times$ & $\times$ \\
{$[15]$} & $\checkmark$ & $\times$ & $\times$ \\
{$[16]$} & $\checkmark$ & $\checkmark$ & $\times$ \\
\hline
\end{tabular}




\subsection{Energy efficiency}

Energy efficiency is an important requirement to consider when designing a WBANenabled IoT MAC protocol, and this requirement focusses on how the energy resources of biosensor devices could be efficiently utilized. A major way by which biosensor devices consume energy is during the channel access process. The energy wasted during this process may be significant due to the likelihood of collisions $[17,18]$ and biosensor devices being battery powered devices may not be able to afford such energy, and hence, for efficient utilization of energy, the usage of energy by the biosensors when trying to access the channel should be minimized through the design of energy-aware MAC protocols that can help to optimize energy during health data communication by reducing the control overhead messages, minimizing the possibility of collisions, optimally allocating channel resources, and by switching the radio into a low power sleep mode when the channel is busy. Also, MAC protocol design for WBAN-enabled IoT should cater for optimizing the level at which energy is being dissipated to prevent energy wastage since the electromagnetic waves radiated by the biosensor devices at high energy levels may damage patients' body and tissue [19]. This means that, the rate at which energy is absorbed by the body must not exceed the specific absorption rate (SAR) given that a WBAN-enabled IoT technology is a special network that is body focused [19].

\subsection{Throughput}

Throughput can be regarded as the successful delivery rate of a health packet over a communication channel. The system throughput aggregate is the total amount of data rates successfully transmitted from all the biosensor devices to the destination device, i.e., from a sender to a receiver, in a WBAN-enabled IoT network $[1,20]$. Because of the scarcity of the communication channel resources and several biosensor devices wishing to access the channel concurrently, collision and/or time wastage issues may be encountered during the exchange of messages. These issues may affect the system achievable throughput, hence, they need to be optimally addressed using efficient MAC protocols. It is important to mention that the throughput requirements are always related to a specific WBAN use case and should be high enough to cater for an application's need and large number of biosensor devices. Moreover, in a WBAN-enabled IoT technology, various factors that include delay-rate, control overhead, collision avoidance, and the utilization of channel may affect the efficiency of the throughput performance. Consequently, these issues need to be carefully considered when designing a MAC protocol for WBAN-enabled IoT applications for efficient health data communications.

\subsection{Latency}

Latency is a measure of the rate of delay which is a function of the time it will take a sent health packet from a biosensor device to be successfully received at the destination device. For critical healthcare systems, like WBAN-enabled IoT, the latency is an important requirement that ascertains how effective is the service provided by such systems. Since WBAN-enabled IoT is a health-focused system, then, health information about patients, especially chronic and critical health conditions, need to be timely and reliably communicated to the appropriate healthcare quarters for prompt actions. Note that, the offered traffic load of the network is a critical underlying factor for timely and 
reliable health data transmission as a very high traffic load may results in a network congestion which causes delay to health data transmission. Likewise, the choice of MAC protocol may also influence the speed of health data communication since it determines the channel access. In the case of WBAN-enabled IoT technology that can only tolerate little or no delay [21], the delay experienced by the biosensor devices while trying to access the channel should be very minimal, and this may vary from WBAN-enabled IoT use cases. But then, the general bound on latency for a non-real time traffic is $250 \mathrm{~ms}$, while that of a real time traffic is around $10 \mathrm{~ms}$ in practice. Hence, the design of MAC protocols for WBAN-enabled IoT technology must support the required latency bound of each WBAN-enabled IoT use case and minimize delays in accessing the channel.

\subsection{Fairness}

Fairness can be described as the ability of various biosensor devices, applications, or users to be able to have equal access to a shared communication channel. Fairness is regarded as an important performance metric in the conventional data and voice networks. It is the minimum number of packets that are transmitted by each biosensor device in relation to the maximum number of packets that are transmitted by any of the biosensor devices in a network. However, in a WBAN-enabled IoT, a biosensor device in a specific time may have more health packets to communicate compared to other biosensor devices and thereby access the communication channel more than the others. Hence, instead of treating individual biosensor device equally, the success of the system is measured by their performance as a whole and each biosensor device fairness becomes unnecessary. To achieve this, the Jain fairness equation can be applied [22]. Due to the heterogeneous nature of WBAN-enabled IoT technology, MAC resource allocation should not always be fair since priorities may be assigned to some biosensor devices, like life critical medical biosensor devices, which may have higher transmission priority than the non-medical devices, during data transmissions.

\subsection{Reliability}

Health packet delivery reliability is another crucial design goal for a WBAN-enabled IoT technology. The guarantee of a successful health packet delivery could be ensured by avoiding overloading biosensor devices with health data, detecting and recovering from health packet drops, and by carefully selecting error-free links. Moreover, there is a typical tradeoff between the overhead traffic control and the reliability level, for instance, per health packet acknowledgment can minimize the recovery time, but constrains its scope at the expense of a high traffic control that may increase energy utilization, increase oneway delay, as well as reduce the link bandwidth effectiveness. Also, the MAC protocol can impact the reliability of health data transfer. For a reasonable reliability, a level above $90 \%$ is typically required for a WBAN-enabled IoT technology packet reception rate.

\subsection{Low complexity}

Another important performance metric that is related to energy efficiency is the necessity for a low complexity operation in WBAN-enabled IoT technology. The reason for this is that such systems are expected to be affordable and simple. Since these systems consist of limited energy biosensor devices, they cannot support computational 
operations that are costly, including complex algorithm. Because of this, low complexity MAC algorithms that provide channel access to the biosensor devices at a low-cost will play a vital role in enhancing the performance of WBAN-enabled IoT technology considering its resource constrained nature.

\section{General overview of MAC protocols used in wireless communications and WBAN-enabled loT technology}

\subsection{WBAN IoT and general wireless communication systems}

A WBAN IoT is a human-focused type of wireless communication system employed in the sphere of healthcare monitoring to collect a patient's health data and send such data to medical physicians in remote locations through a computer network [2]. On the other hand, a general wireless communication system is a type of wireless network deployed in an application domain other than healthcare for data communication purposes. An example of a typical general wireless communication system includes a wireless sensor network (WSN). It is important to mention that a WBAN IoT is different from other types of wireless communication systems in terms of application focus, requirements, and characteristics. For example, a WBAN IoT focuses more on quality-of-service (QoS) requirements like energy efficiency, latency, data rate, network heterogeneity, and reliability, while a general wireless communication system like WSN focuses more on power conservation [23]. Also, both WBAN IoT and WSNs are differentiated in terms of the employed sensor type and environment. For instance, WBAN IoT environment is typically highly dynamic in nature as the patient wearing a WBAN IoT is mobile, while a WSN environment is typically static in nature since most of the application areas of a WSN is stationary. These differences have orchestrated the need for different QoS considerations in the MAC designs for both WBAN IoT and WSN.

\subsection{General overview of MAC protocols in wireless communication systems}

The MAC layer is an important building block in the wireless communication systems' protocol stack model which comes immediately after the PHY layer. It is used to manage the allocation of the communication channel. For example, the MAC layer of the WBAN-enabled IoT technology is responsible for managing and controlling the times when a set of biosensor devices can access a shared communication channel for communication purposes, and its channel resource allocation process may be optimized to satisfy the stringent network QoS requirements of WBAN-enabled IoT technology in terms of energy efficiency, throughput, fairness, and latency [11]. For this purpose, an efficient MAC protocol for WBAN-enabled IoT technology should be aware of different WBAN QoS requirements and capable of supporting various health data types, such as an emergency health data, normal health data, continuous health data, periodic, and bursty health data without compromising the QoS requirements of the application. Furthermore, a good MAC protocol must be energy efficient because of the energy resource scarceness issue related to wireless communication systems such as the WBAN-enabled IoT technology. Note, the major energy wastage issues often experienced by the biosensor devices when accessing the channel for communication purposes are collisions and idle listening. Therefore, these issues must be addressed for a WBAN-enabled IoT technology to be energy efficient. 
Additionally, due to the ad hoc means by which biosensor devices gain access to the radio channel, there are two major problems they mostly experience that causes collisions, latency, and channel under-utilization. The problems are the hidden-terminal problem and exposed-terminal problem. A hidden-terminal problem is experienced when two biosensor devices sense a channel to be free due to their inability to properly hear each other, unlike an AP which is fully aware of all the network devices, and simultaneously transmit their individual sensory health data to an AP. This problem often leads to collisions. To resolve this issue, a biosensor device needs to first accurately listen to the communication channel to identify when it is in a busy or idle mode before sending its health packet. An exposed-terminal problem occurs when a biosensor device that wish to access the channel mistakenly sense that there is an ongoing transmission and therefore denied an access to the channel or needlessly differs its transmission. The hidden-terminal problem wastes energy resources through collisions, while the exposed-terminal problem causes delay, time-resource wastage, and inefficient channel utilization or bandwidth resources under-utilization.

To deal with the various MAC layer related issues that may potentially waste the network resources, insights into different MAC protocols are essential. Because of this, this study classifies the existing MAC protocols into four major categories that include the contention-free, contention-based, hybrid MAC, and the MAC protocols that are specific to WBANs in Fig. 2, and a general overview of these protocols and their suitability for WBAN-enabled IoT technology are presented in this section since they are fundamental to developing new protocols.

\subsubsection{Contention-free MAC protocols}

This section briefly presents the primary details about the contention-free MAC protocols for insights into their operation. The contention-free MAC protocols are regarded as schemes that could coordinate the communication between the biosensor devices in the context of a WBAN-enabled IoT technology in a well-ordered manner. In this category, the available communication channel is shared between several biosensor devices using a scheduling method to circumvent collisions, and the biosensor devices will not need to compete with one another before accessing a communication channel [24]. With this type of schedule, the protocols attempt to minimize energy utilization by organizing the biosensor devices in a common scheduling manner. This

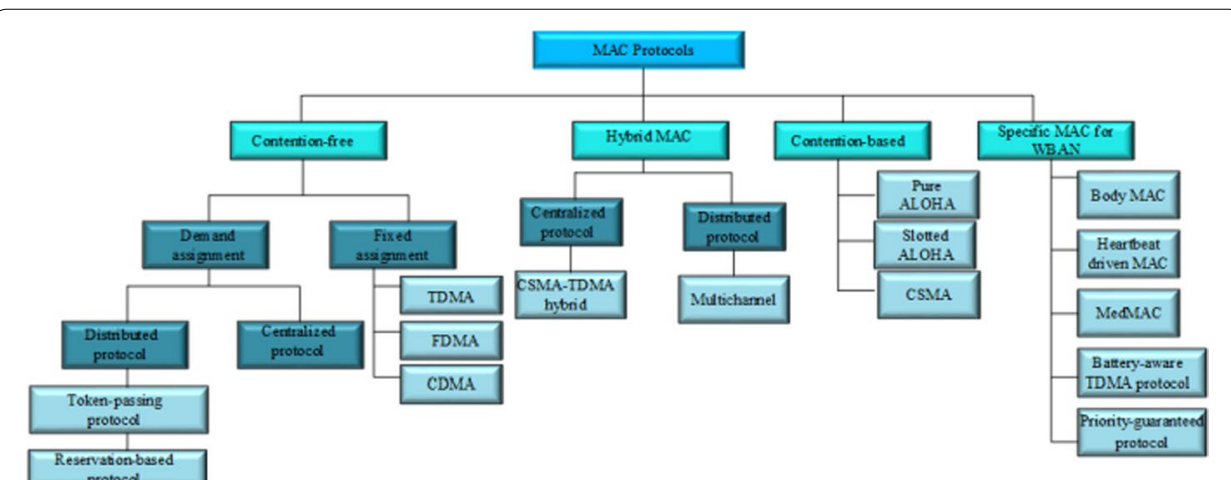

Fig. 2 Classification of MAC schemes for the WBAN-enabled loT technology 
help the protocols to clarify the biosensor devices that should access the communication channel at any period of time, thereby, avoiding unnecessary idle listening, overhearing, and collisions.

The contention-free schemes may be classified into two main groups, including the fixed assignment and dynamic (or demand) protocols [24], and are discussed in the subsequent subsections.

4.2.1.1 Fixed assignment protocols The protocols in this group divides the available resources among the biosensor devices in the context of a WBAN-enabled IoT technology so that the assigned resources are in long term, i.e., the assignment is for longer durations. An individual biosensor device exclusively utilizes its allocated radio resources without having to compete with other biosensor devices. Examples of the protocols in this category are the time-division multiple access (TDMA), frequencydivision multiple access (FDMA), and the code-division multiple access (CDMA). The FDMA works by dividing radio spectrum into frequency sub-bands, while each frequency sub-band is separated by a guard band to prevent interference among adjacent channels, and allocated to each individual biosensor device in a WBAN-enabled IoT technology. The allocated band is large enough to take in the signal spectral transmissions to be broadcasted [24] and may enhance the throughput and latency performance of a network. The spectral efficiency of the FDMA scheme can be determined using (1) according to [28] as:

$$
\eta_{\mathrm{s}}=\frac{C_{\mathrm{s}} T_{\mathrm{c}}}{S_{\mathrm{B}}} \leq 1
$$

where $\eta_{\mathrm{S}}$ is the spectral efficiency, $C_{\mathrm{S}}$ is the channel spacing, $T_{\mathrm{C}}$ represents the total number of the channels' traffic, and $S_{n}$ represents the bandwidth of the system.

Unfortunately, the FDMA protocol suffers from bandwidth wastage issue because of the guard band provision and the capability of each biosensor device to have a dedicated frequency band. Other potential issues include lack of support for biosensor devices with heavy traffic, scalability concern, high-cost hardware implementation issue. Unlike the FDMA, the TDMA protocol allows biosensor devices to share a single communication channel without any form of interference. It achieves this by dividing the communication channel into different time slots and allocate a particular time slot to an individual biosensor device at a specific time. Consequently, each biosensor device transmission is scheduled via a controller (such as a central AP) and their achieved in a round robin scheduling manner. It is crucial to mention that the TDMA protocol can operate either as a narrowband or a wideband TDMA system. In the narrowband TDMA, spectral efficiency can be calculated in (2) given by [28] as:

$$
\eta_{\mathrm{S}}=\left(\frac{\left(\tau T_{\mathrm{S}}\right)}{T_{\mathrm{fr}}}\right)\left(\frac{U_{\mathrm{B}} U_{\mathrm{N}}}{S_{\mathrm{n}}}\right)
$$

where $\tau$ is the time slot duration, $T_{\mathrm{s}}$ is the time slot number in a frame, $T_{\mathrm{fr}}$ is the frame duration, $U_{\mathrm{B}}$ denotes the user's bandwidth in a given time slot, and $U_{\mathrm{N}}$ denotes the number of users in the system sharing a time slot. While, the spectral efficiency of the wideband TDMA can be expressed in (3) as: 


$$
\eta_{\mathrm{S}}=\frac{\tau T_{\mathrm{S}}}{T_{\mathrm{fr}}}
$$

This protocol requires a less energy since a biosensor device is only in an active state when it is scheduled to send or receive a health packet, thus conserving its energy when not in an active state. The biosensor devices in a TDMA system often remains in a sleep mode when the slot is not active, thus circumventing the energy wastage issue related to an idle state and overhearing [25]. Also, there are no collision and retransmission issues in a TDMA protocol. As a consequence, a TDMA protocol supports energy efficiency and can be applied to WBAN-enabled IoT technology. This protocol has been exploited in WBAN systems, and some good examples are [26, 27]. In CDMA, multiple biosensor devices can be enabled to transmit their health packets concurrently with a minimal interference. This could be achieved by providing a unique code to each biosensor device to separate their transmissions. Because of the CDMA transmission nature, it employs a forward error correction (FEC) method to recuperate from any possible interference experienced between the biosensor at the receiver [28]. Some major issues with CDMA are delay, interference, and channel allocation issue. Hence, this protocol may be regarded as energy efficient and may not be a good candidate for WBAN-enabled IoT technology. But then, the constraints of these protocols could be improved upon by combining the protocols together.

\section{Summary of the fixed assignment MAC protocols}

Among the reviewed fixed assignment protocols, most research works have employed the TDMA scheme compared to the CDMA and the FDMA schemes due to the potentials of the schemes, i.e., CDMA and FDMA, to increase the energy requirement and the implementation cost of the biosensor devices. Also, in general, the fixed assignment protocols are considered to be disadvantageous because of their inability to reassign slots that belongs to one biosensor device to other biosensor devices if they are not needed in each time frame. Another disadvantage of the fixed assignment protocol approaches if considered for the WBAN-enabled IoT technology is that the generation of schedules for the entire system may be a demanding task, and this could make schedules to require constant modifications each time the traffic features in the network changes or network topology changes. For the purpose of clarity, Table 2 presents a qualitative analysis of the TDMA, FDMA, and CDMA protocols.

4.2.1.2 Demand/dynamic assignment protocols The protocols in this group exclusively assigns the available resources including the radio channels to the biosensor devices in a short-term form. Technically, the main aim of the demand assignment protocols

Table 2 Qualitative analysis of TDMA, FDMA, and CDMA protocols [25-28]

\begin{tabular}{llll}
\hline Parameters & TDMA & FDMA & CDMA \\
\hline Energy efficiency & High & Low & Low \\
Latency & Moderate & Moderate & Moderate \\
Throughput & Moderate & Low & Moderate \\
Reliability & High & Moderate & Moderate \\
Channel utilization & Moderate & Low & High \\
\hline
\end{tabular}


is to enhance the utilization of the radio channel by assigning the channel to the competing biosensor devices in an optimal or near-optimal manner. Compared to the fixed assignment techniques that may exclusively allocate channel to the biosensor devices of a WBAN-enabled IoT network in a pre-determined manner without considering their communication requirements such as the size of the health packet. The demand assignment techniques on the other hand disregard the biosensor devices that are in an idle state and only put into consideration the biosensor devices that are prepared to transmit their health packets. It is worthy of note to mention that in the existing literature the dynamic assignment protocols proposed are mainly based on a TDMA approach and mostly focus on adapting slots number or reallocation of slots based on the number of the biosensor devices that are active as well as their traffic intensity [29].

The demand assignment schemes could be grouped into two main classes including the distributed and the centralized schemes. In the centralized protocols, the biosensor devices broadcast requests for the allocation of a bandwidth to a central device and the request may be either accepted or rejected. However, depending on the protocol characteristics, the necessity to request an access to the radio channel may cause a delay in the transmission of the health packets. Moreover, a logical control medium apart from the health packet channel may be needed by the competing biosensor devices to dynamically request for an access to the radio channel. For the distributed protocols examples are the token-passing and the reserved-based methods. In the token-passing protocols, controllers are needed to pass a polling request to one another in a round-robin manner through a special small frame known as a token. The token is rotated between the biosensor devices and is arranged in a logical ring on the topmost part of a broadcast channel. The controller is only allowed to send a health packet when it has a token. An example of a WBAN system that adopted this protocol is [30] where the authors considered a token-based dynamic MAC protocol for a WBAN system. The reserved-based method allows fixed time slots to be employed for a future access reservation to a radio channel. For instance, a biosensor device may indicate its wish to send a health packet by switching a reservation bit in a static location [21]. An example of a research wok on a reservation-based dynamic method is [31].

\section{Summary of the demand/dynamic assignment MAC protocols}

The demand/dynamic assignment protocols are examples of the contention-free techniques where the radio channel is assigned to a biosensor device on demand, unlike the fixed assignment protocols that is on a fixed basis. The main aim of the demand assignment protocol is to enhance the radio channel utilization through the assignment of the radio channel capacity to competing biosensor devices in an optimal or near-optimal manner. The protocols in this group technically uses a network control scheme to grant the competing biosensor devices access to the radio channel. Moreover, a logical control radio channel, apart from the health packet radio channel could be required by the competing biosensor devices to request access to the radio channel.

\subsubsection{Contention-based MAC protocols}

The contention-based MAC protocols are types of schemes that allows many biosensor devices to contend with one another in an uncoordinated way to gain an access to a radio channel. Unlike the contention-free protocols, they do not depend on a transmission 
schedule, but rather employs some other methods, like the contention-based algorithms, to resolve the contention issue that may potentially arise. The contention-based protocols seem to be promising because of their advantages in contrast to the contention-free protocols according to [32]. For instance, the contention-based techniques are flexible, robust and simple, they do not require the channel state information to be shared, maintained, or saved, and therefore enables them to quickly adapt to the changes in the traffic characteristics or the changes in a WBAN-enabled IoT network topologies, and also, they do not require too much infrastructure support such as a clock for synchronization purposes. However, the aforementioned advantages of the contention-based protocols over the contention-free protocols typically come with a weakness at the expense of collision due to the fact that a biosensor device may use a trial and error method for accessing the radio channel when attempting to send a health packet. The devices can also experience an unfairness problem in channel access. The common examples of the protocols that falls under this category are referred to as the random access protocols such as the CSMA and the ALOHA protocols.

The ALOHA was designed to typically allow each transmitter, i.e., biosensor device, in a communication system, like WBAN-enabled IoT technology, to transmit its health packet without controlling the transmission. It utilizes an acknowledgement or a retransmission policy to handle a possible collision problem. This protocol has two variants, namely the pure ALOHA protocol and the slotted ALOHA protocol. In a pure ALOHA protocol, whenever a biosensor device has a new health packet to send, it simply transmits the health packet immediately. Afterward, a biosensor device finds out by listening to the channel if the transmission was successfully received at the receiver, such as an AP, or has experienced a collision. In order to detect if there is a collision or not, the receiver will have to send an acknowledgement message for a successfully received health packet, while the transmitter considers the absence of a non-acknowledgement message as an indication that a collision has occurred. Considering a transmission scenario, according to [33], packets can be generated using a Poisson distribution, the probability of the Poisson arrivals of the packets can be expressed in (4) as:

$$
P_{m}(t)=\frac{(\lambda \times t)^{m} \times e^{-\lambda \times t}}{m !}, m=0,1,2, \ldots
$$

where $m$ is the arrivals of packets generated in a finite time arrival interval $t$, and $\lambda$ is the arrival rate. Additionally, the probability that a packet is successfully transmitted is the probability that there are no extra packets transmission in the vulnerable time. A vulnerable time in pure ALOHA implies the time in which no transmission is expected to occur so as to avert a collision. In pure ALOHA, a vulnerable time is expressed in (5) as:

$$
V_{\mathrm{T}}=2 \times T_{\mathrm{pk}}
$$

where $V_{T}$ is the vulnerable time and $T_{p k}$ is packet transmission time. Since the probability of a successful transmission would imply that there is no arrival of packets during the vulnerable period, i.e., no occurrence of a collision, then the probability of a successful packet $\left(P_{\text {success }}\right)$ transmission is defined in (6) as: 


$$
P_{\text {success }}=G \times \exp ^{-2 G}
$$

where $G$ is the offered traffic. If a collision eventually occurs, then the transmitter backoff for some random period of time. The back-off time is regarded as the time required for a transmitter involved in a collision to wait before trying to resend the health packet again $[33,34]$. Some issues associated with the pure ALOHA protocol are energy efficiency, channel utilization, and throughput $[35,36]$.

To improve upon this protocol, a slotted ALOHA protocol was introduced in 1972 by Roberts [37]. Unlike the pure ALOHA protocol, the slotted ALOHA does not allow the transmission of health packets whenever a biosensor device has a health packet to transmit. Instead, a biosensor device that wants to transmit a health packet has to wait till the beginning of a time slot. The available time resource is divided into time slots, and each slot is sufficient enough to accommodate the maximum length of a health packet. In addition, the biosensor devices are synchronized in such a way that each biosensor device knows the beginning of the slots, such a synchronization is achieved through an additional overhead. The possibility of a collision occurrence may also be envisaged in the slotted ALOHA protocol when two biosensor devices attempt to transmit their health packets at the start of a time slot [38, 39]. Also, the vulnerable time is lowered by half compared to the pure ALOHA and is given in (7) as:

$$
V_{\mathrm{T}}=T_{\mathrm{pk}}
$$

While, the probability of a successful packet transmission in slotted ALOHA is expressed in (8) as:

$$
P_{\text {success }}=G \times \exp ^{-G}
$$

The CSMA protocol utilizes a carrier sensing (CS) method for assigning an idle channel to biosensor devices in a CSMA-based WBAN-enabled IoT technology [40]. In this protocol, a biosensor device that wish to transmit senses the carrier, i.e., channel, to know if there is an ongoing transmission so as to minimize the issue of collision. Therefore, vulnerable time in the CSMA protocol is equal to the propagation time. If the biosensor device found the channel to be busy, it will either wait or reschedule its health packet transfer process based on an amount of period that is determined by the used algorithm [41]. But then, if the channel is found idle, the biosensor device starts its transmission immediately. This protocol typically suffers from two major problems, including the hidden-terminal and exposed-terminal problems. These problems are promoters of collisions, time-resource wastage, and inefficient channel utilization.

Currently, there are different variants of the CSMA scheme available in literature to provide possible solutions in a situation where a transmission channel is found busy, and the examples of such variants are the non-persistent CSMA, the 1-persistent CSMA, and the p-persistent CSMA [41-43]. Considering a transmission scenario, according to [41-43], the throughput efficiency of a non-persistent and a 1-persistent CSMA can be calculated using (9) and (10) respectively as: 


$$
\begin{aligned}
& S=\frac{G \exp ^{-a G}}{G(1+2 a)+\exp ^{-a G}} \\
& S=\frac{G \exp ^{-G} x(1+G)}{G+\exp ^{-G}}
\end{aligned}
$$

where $G$ is the packet load and $a$ is the propagation normalized delay.

Because of the collision concern related to the CSMA protocol, the evolution of a CSMA protocol with a collision avoidance (CA), i.e., CSMA/CA, is a promising protocol [44]. In a CSMA/CA protocol, a biosensor device that is ready to transmit its health packets first listens to the radio channel to ensure it is free before transmission would take place. If the radio channel is found idle, the health packet would be transmitted, else the biosensor device will back-off for a random period of time and later listens again to know if the channel is idle. Note, depending on the back-off algorithm employed, if the channel is free when back-off counter gets to zero (i.e., 0 ), then the biosensor sends its health packets, but if the channel is busy when the back-off counter gets to 0 , then the back-off is reset again and this process is repeated continuously until the channel is free [45]. It is noteworthy to mention that the CSMA/CA protocol prevents a collision from happening before transmission by first sending a request to send (RTS) message to the $\mathrm{AP}$, and the AP responds back with a clear to send (CTS) message. Afterward, health packet transmission can commence, and if an acknowledgment message is not received from the AP after transmission, the biosensor device assumes the occurrence of a collision and try to resend the health packet again until an acknowledgement message is received [44].

\section{Summary of the contention-based MAC protocols for WBAN-enabled IoT}

In practice, the slotted ALOHA protocol has been established to have a maximum throughput efficiency of about 37\% compared to the pure ALOHA protocol which has a maximum throughput efficiency of about $18 \%$ when $G=1$, and is also energy efficient [39]. Consequently, the slotted ALOHA protocol is envisaged to be a promising candidate that can be explored as an energy efficient MAC protocol in WBAN-enabled IoT technology. However, the exploitation of this protocol requires a careful design due to the inherent collision issue related to the protocol. While, the 1-persistent CSMA has a maximum throughput efficiency of about $50 \%$ and the non-persistent CSMA has a maximum throughput efficiency of about $90 \%$ when $G=1$ [44]. As a consequence, the CSMA protocols are more promising for a potential utilization in WBAN-enabled IoT technology compared to the ALOHA protocols. However, because of the contending nature of these protocols for channel utilization, they typically suffer from latency, reliability, and energy efficiency issues. These concerns make them to be disadvantageous in WBAN-enabled IoT technology, but then, their shortcomings can be carefully optimized to make them productive. For the purpose of clarity, a qualitative analysis of the pure ALOHA, slotted ALOHA, and the CSMA protocols are presented in Table 3. 
Table 3 Qualitative analysis of pure ALOHA, slotted ALOHA, and CSMA protocols [39-45]

\begin{tabular}{llll}
\hline Parameters & Pure ALOHA & Slotted ALOHA & $\begin{array}{l}\text { CSMA (1-persistent } \\
\& \text { non-persistent) }\end{array}$ \\
\hline Energy efficiency & Moderate & High & Low \\
Latency & Moderate & Moderate & High at light loads \\
Throughput & Low & Moderate & High \\
Reliability & Low & Moderate & Moderate \\
Channel utilization & High & Moderate & Moderate \\
\hline
\end{tabular}

\subsubsection{Hybrid MAC protocols}

Hybrid MAC protocols are formed as a result of combining or mixing the contentionfree MAC protocols and the contention-based MAC protocols together. The contention-based protocols have the ability to adapt to changing network easily and they are also suitable for low load networks compared to the contention-free protocols, while the contention-free protocols are slightly suitable in terms of channel utilization at high loads. For this reason, harnessing the benefits of these two classes of protocols so as to off-set their limitations is important [46]. As a consequence, hybrid MAC protocols are potential methods for developing efficient MAC protocols for WBAN-enabled IoT technology. The idea of mixing protocols together has been explored and applied to WBANs in literature, good examples are $[4,47,48]$. In [4], a hybrid MAC protocol was proposed by mixing the CSMA/CA and the TDMA protocols (i.e., CSMA/CA-TDMA) to improve the energy efficiency of WBAN biosensor devices as well as extend their lifespan. For the purpose of evaluation, the protocol was compared with the Quasi-SleepPreempt-Supported (QS-PS) and the channel-aware polling-based MAC protocols using 9 biosensors, a guard time interval of $0.00003 \mathrm{~s}$, a transmission rate of $5 \mathrm{Mbps}$, and a transmission probability of 0.8. The result of the simulation is presented in Fig. 3 .

From Fig. 3, we noticed that the more the biosensors in the network were increased from 3, 5, 7 to 9 biosensors, the more the energy consumed for the three MAC protocols. However, the CSMA/CA-TDMA protocol was observed to be more energy efficient compared to the other protocols. For example, when the network was simulated with 5 transmitting biosensors, the energy consumed using the CSMA/CA-TDMA protocol was about $236 \mathrm{~mJ}$, while about $259 \mathrm{~mJ}$ and $245 \mathrm{~mJ}$ energy were consumed using the QS-PS and the CPMAC protocols, respectively. This implies that the CSMA/CA-TDMA was able to achieve about 15\% energy reduction than the QS-PS and about $10 \%$ energy reduction than the CPMAC. These improvements could be attributed to the hybrid method employed, i.e., combining the advantages of the CSMA/CA and the TDMA protocol as well as the division of the biosensors into different states, for instance, the awaiting orders state. In addition, a significant amount of energy was saved by allocating most of the overhead transmission at the personal station. However, the scalability of the system still remains a concern. The authors of [47] presented a hybrid multi-channel MAC (HMC-MAC) protocol for WBANs by mixing the CSMA/CA and TDMA protocols to mitigate the likelihood of inter-WBAN interference, i.e., collisions. The proposed protocol enabled multiple transmissions concurrently on different radio channels which resulted to a high throughput with a low energy consumption as well as a reduced collision occurence with the help of a channel selection scheme. For the purpose of 


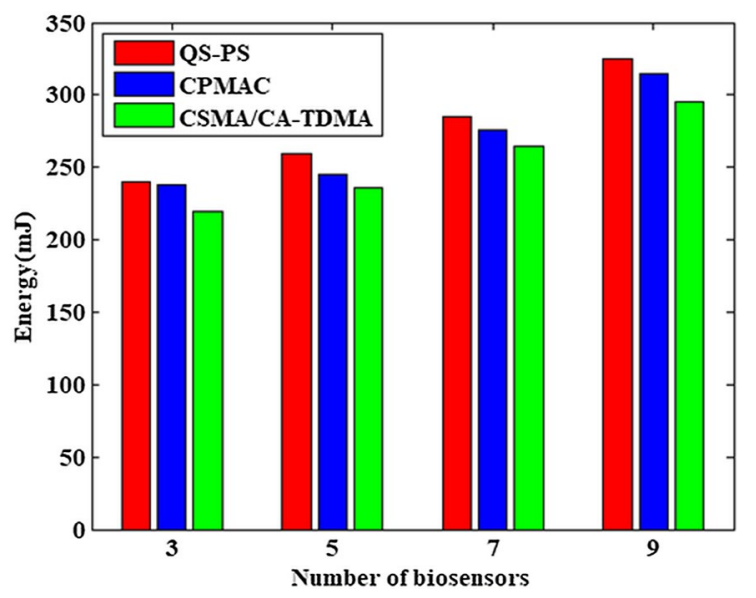

Fig. 3 Energy versus number of biosensors

performance comparison in terms of energy efficiency, the HMC-MAC was compared with a traditional multi-channel MAC protocol (MC-MAC) using a total number of 10 biosensors that are classified into high priority (HP) and low priority (LP) per WBAN with an arrival rate of 10 packets/secs and a data rate of $250 \mathrm{kbps}$. The outcome of the simulation is presented in Fig. 4.

We realized from Fig. 4 that the HMC-MAC protocol is advantageous in the context of energy savings. For example, when 3 WBAN systems were considered, we noticed that the HMC-MAC with HP has a total energy consumption of about $10 \mathrm{~J}$, while the MC-MAC with HP has about $60 \mathrm{~J}$ and the HMC-MAC with LP has a total energy consumption of about $150 \mathrm{~J}$, while the MC-MAC with LP has $200 \mathrm{~J}$. This is indicative that the HMC-MAC with $\mathrm{HP}$ was able to achieve a $25 \%$ reduction in energy consumption than the MC-MAC with HP. This improvement was as a result of the channel selection algorithm that was introduced to prevent the occurrence of collisions among neighboring WBANs, thereby improved energy efficiency. Also, the authors of [48] considered a hybrid MAC protocol for WBANs by combining the CSMA/CA and TDMA protocols to mitigate collisions issue. The proposed protocol enabled multiple transmissions concurrently on different radio channels which resulted to a high throughput as well as a reduced collision with the help of a channel selection scheme.

However, in the literature, it is vital to note that there are some limitations that could be attributed to a hybrid MAC protocol, for instance the scalability issue and message control overhead concern. A hybrid MAC protocol scalability issue can be improved by exploiting a hybrid protocol based on the CDMA or FDMA protocol, but then, the major limitation of the FDMA is the expensive hardware as well as that of the CDMA in terms of complex operations. Also, power control requirement is another limitation of these protocols. Thus, in the context of WBAN-enabled IoT technology, the TDMA based hybrid MAC protocols have promising potentials compared to that of the FDMA and CDMA protocols. Because of this, a work carried out in [49] combines the TDMA and FDMA protocols to offer two channels when transferring packets from the devices to an AP. The combination of the TDMA and FDMA protocols was used to minimize energy utilization with a collision-free packet transmission. 


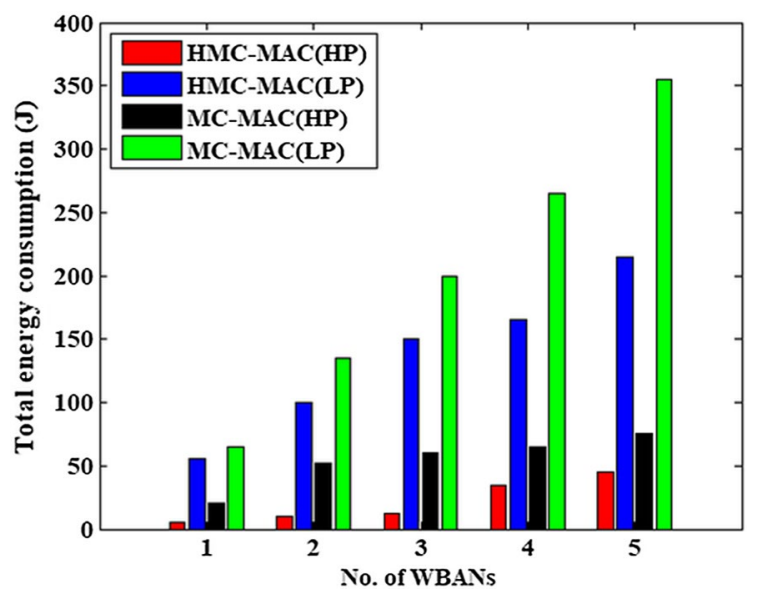

Fig. 4 Total energy consumption

\subsubsection{WBAN-specific MAC protocols}

The MAC protocols that falls under this category are designed to address the specific requirements of the WBAN communication systems. At present, there are a number of MAC protocols proposed in literature for WBANs, for insights into these protocols, this study has considered some of the state-of-the-art MAC protocols that specifically targets WBANs. Examples are the BodyMAC protocol, the Heartbeat-driven MAC protocol, the Medical MAC (MedMAC) protocol, the battery-aware TDMA protocol, the priority-guaranteed MAC protocol, and so on. Also, this study covers the advantages and disadvantages of these prominent MAC protocols, as well the protocols are discussed with focus on how to address energy inefficiency that could be caused by issues like idle listening, collision, and packet overhead. All these are considered in the subsequent subsections.

4.2.4.1 BodyMAC protocol The BodyMAC protocol is one of the prominent protocols that have been specifically designed for WBAN use cases [50], and is based on a TDMA approach. The BodyMAC protocol has its channel bounded by the superframe structure of the TDMA with UL and DL sub-frames. The essence of this protocol is to optimize the power consumption of WBANs. Because of this, the BodyMAC protocol provides a flexible bandwidth allotment to enhance energy efficiency through the reduction of packet collisions, transmission time, packet overhead, as well as the reduction of idle listening. This protocol works in a way that the biosensor devices go into a sleep mode when not active, i.e., when they have no data to transmit, and this help to conserve the battery power resource.

Note, the MAC frame structure of the BodyMAC is divided into three segments, namely the beacon, DL, and the UL. The beacon period is responsible for the MAC layer synchronization and also describes the MAC frame structure. The DL segment is reserved for communication from the AP to the biosensor devices to accommodate on-demand traffic. The UL segment is used for the communication from the biosensor devices to the AP. It has two phases that include the contention access phase (CAP) 
which employs the CSMA/CA scheme and the contention-free phase (CFP) as shown in Fig. 5 [50].

In the CAP process, the biosensor devices contend for the transmission of control packets to the AP for guaranteed time slots (GTS), and in the CFP process, the AP is responsible for controlling the allocation of the GST to the biosensor devices in order to prevent a collision occurrence. It is important to mention that the communication in the CFP is helpful in improving energy efficiency. But then, for the UL segment in the CAP, the CSMA/CA protocol employed results to a high energy consumption because of the collision issue and the clear channel assessment (CCA). Also note that the MAC layer synchronization mode of this protocol is energy expensive as a significant amount of energy is expended for the exchange of beacon signals [13].

For performance evaluation purposes, simulation investigations were performed on the BodyMAC protocol, the IEEE 802.15.4 MAC protocol, and the CSMA/CA protocol with 24 biosensors and a data rate of $8 \mathrm{kbps}$ in the context of energy conservation and the obtained simulation results are presented in Fig. 6 [50].

From Fig. 6, we could deduce that the BodyMAC has a less energy consumption level when compared with the CSMA/CA and the IEEE 802.15.4 MAC protocols. For instance, when time was set to $16 \mathrm{~s}$, the energy consumed by each biosensor was about $0.5 \mathrm{~J}, 0.17 \mathrm{~J}$, and 0.14 J when the CSMA/CA, IEEE 802.15.4, and BodyMAC protocols were applied, respectively. This reveals that the BodyMAC protocol is advantageous in terms of energy savings and has performance gains of about $36 \%$ and $18 \%$ over the CSMA/CA and the IEEE 802.15.4 MAC protocols respectively. These improvements are due to the exploitation of a sleep mode mechanism which helped the biosensors to be in an active mode only when they have packets to send unlike the CSMA/CA protocol where the biosensors will have to be in an idle mode before they can send their packets, while the IEEE 802.15.4 MAC employed the GTS which may waste the energy of the biosensors when they are inactive. But then, packet collision issue still remains a concern.

4.2.4.2 Heartbeat-driven protocol The heartbeat-driven protocol is another important protocol that was developed for WBAN applications to improve energy efficiency in [51]. The heartbeat-driven protocol is also based on a TDMA protocol and was designed for WBANs to use the heartbeat rhythm information to perform synchronization instead of a beacon. Heartbeat rhythms are natural phenomenon in the body of every human being and is observable in different bio-signals. It allocates dedicated slots time to each individual biosensor device in order to guarantee a collision-free data transmission. It is worthy of note to mention that the heartbeat-driven protocol is aimed at improving the

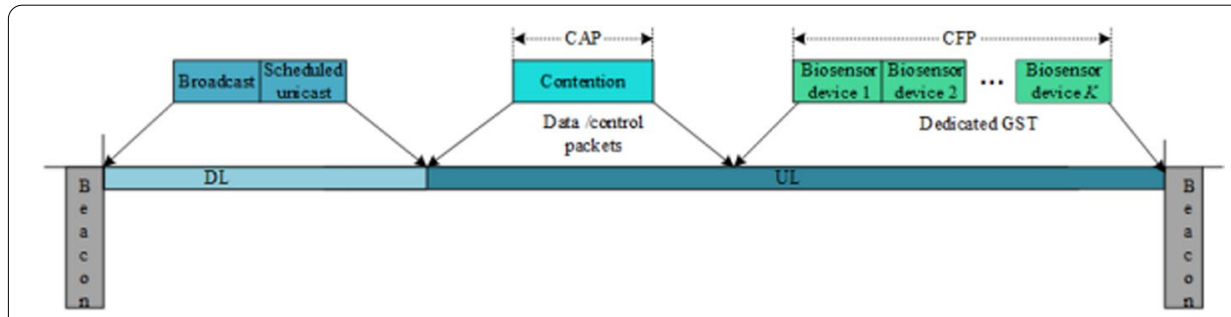

Fig. 5 A typical BodyMAC frame structure 
energy efficiency of the biosensor devices by avoiding the need for a periodic synchronization. The consequence of this is that, time synchronization can be achieved through the heartbeat rhythm without turning on their radios for the reception of periodic timing messages from a centralized controller so that the time synchronization energy cost can be eliminated as well as prolong the network lifespan. The protocol proposed in [51] for body sensor networks use a synchronization recovery policy with two resynchronization methods. The simulation results of [51] revealed that the heartbeat MAC protocol has the potential of prolonging the network lifetime. However, this solution has a high energy consumption and high delay rate problems which could make it unsuitable in emergency conditions [13].

4.2.4.3 MedMAC protocol In literature, a MedMAC protocol has been proposed for WBANs to reduce energy consumption and improve the channel access method [52]. The MedMAC adopts a TDMA scheme for allocating time slots for transmission to the biosensor devices. The allocated time slots vary in length and also based on the biosensor devices requirements. As shown in Fig. 7, the MedMAC protocol employed a multisuperframe structure that uses beacons for synchronization purposes [52].

Additionally, for communication with low data, network initialization, and emergency traffic, an optimum contention period is used. Also, the MedMAC employed a unique GST for each biosensor device to prevent a collision occurrence. The MedMAC was compared with the IEEE 802.15.4 MAC in [52] to investigate its energy consumption performance. To this end, simulations were carried out by grouping the biosensor devices into class 0 and class 1 applications. The biosensor devices in class 0 contains 3 devices and are used for monitoring health/fitness, including temperature, pulse, and respiration. While, an EEG system with 24 biosensors was considered in class 1 with a latency of $<250 \mathrm{~ms}$ and a bit rate of $250 \mathrm{kbps}$. Figure 8 shows the simulation results of the energy consumed by the 24 EEG biosensors.

We noticed from Fig. 8 that as the number of the biosensors increases the energy consumed by each of the biosensors also increase when the IEEE 802.15.4 MAC protocol was applied, but when the MedMAC protocol was applied there was no

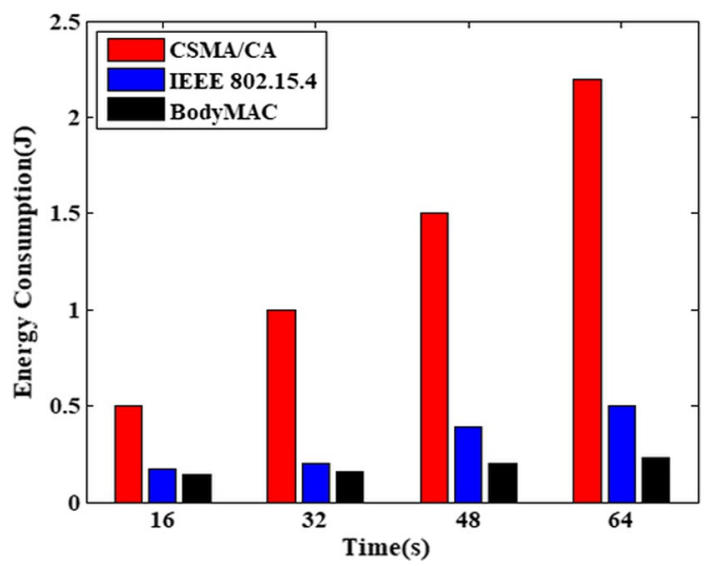

Fig. 6 Energy consumption versus time 
variation in the energy consumed by each of the biosensors as they increase. For example, when there are 4, 8, and 12 biosensors in the EEG system the average energy consumption using the IEEE 802.15.4 was around $5 \mathrm{~mW}, 9 \mathrm{~mW}$, and $13 \mathrm{~mW}$ respectively, while that of the MedMAC was about $0.1 \mathrm{~mW}$ and did not vary as the number of the biosensor increases. This indicates that the MedMAC was able to achieve about $10 \%$ energy consumption reduction than the IEEE 802.15.4. This improvement was due to the dedicated time-slot that was allocated to each of the biosensors for transmission and this helped to prevent collisions occurrence unlike the IEEE 802.15.4 MAC that employed the CSMA/CA protocol to reduce the number of collisions. But then, collisions may be experienced in the retransmission of the packets by the biosensors in a CSMA/CA network, resulting into energy wastage. On the other hand, the MedMAC only provide support for a low data traffic and this could make it unsuitable for modern WBANs especially for the implantable and wearable sensors which requires a high data rate.

4.2.4.4 Battery-aware TDMA protocol A battery-aware TDMA protocol is another protocol designed to improve the WBAN's network lifetime by employing a cross-layer method [53]. This protocol operates like the IEEE 802.15.4 beacon mode where the biosensor devices have to periodically listen to the beacons from the AP, i.e., the coordinator. The design of this protocol incorporates several factors, including the battery electrochemical properties, the packet queuing features, and the time-varying wireless fading channel. This protocol exploits the time-axis region to partition its operation processes into three parts, namely the inactive period, active slot time, and the beacon slot [53]. In order to support the various WBAN application requirements, the frame length is adaptive and could be changed. At the beginning of the beacon phase, a periodic wakeup scheme was introduced to prevent the biosensor devices from an unnecessary idle listening. In addition, a dedicated slot time $T_{\mathrm{S}}$ is allocated to each biosensor device for data transmission in the active phase after it receives a beacon. For the inactive phase, the biosensor devices go into a sleep mode in order to save energy. The battery-aware TDMA protocol prolongs the lifespan of the biosensor devices and the GTS allocated to each biosensor device enhances packet delivery in a timely manner and improves reliability. The limitations of this solution include the inability to hold or retain packets in the buffer for long which could result to a high rate of packet

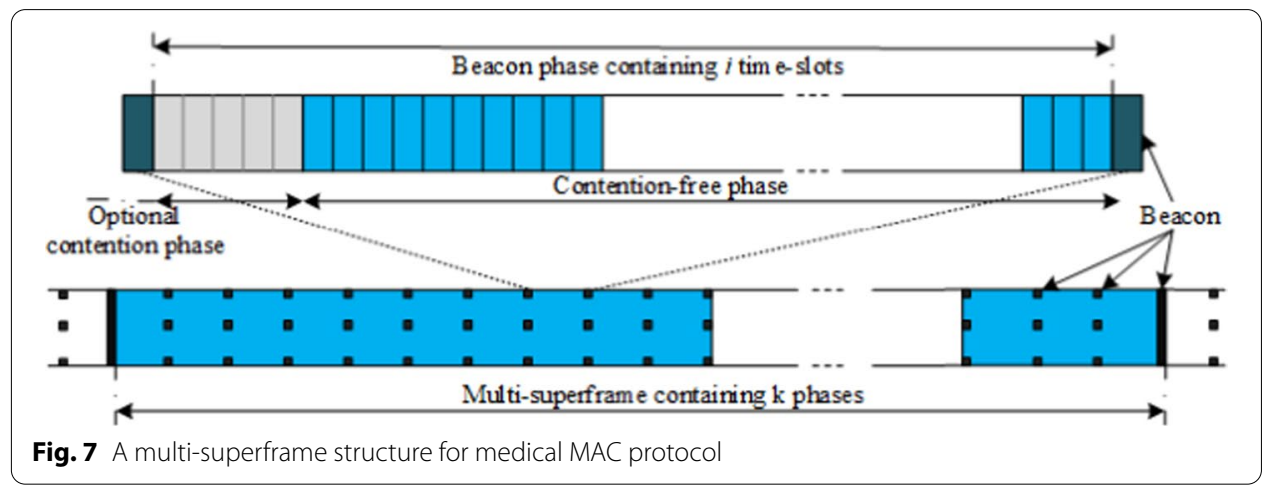




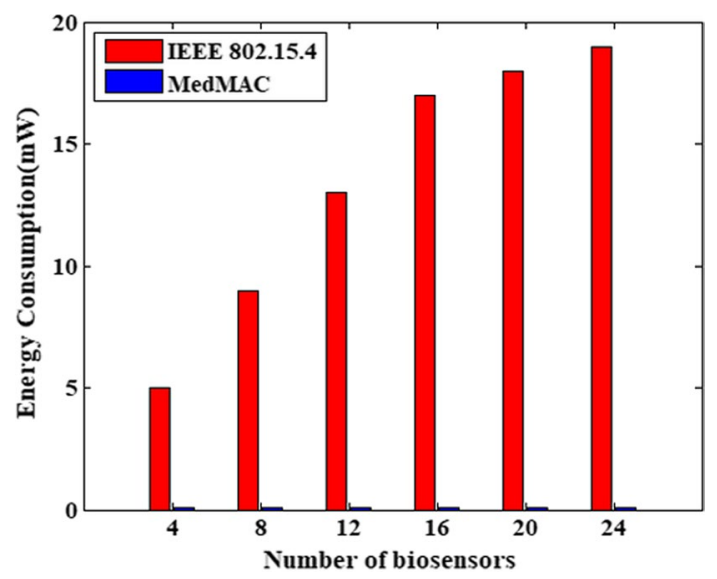

Fig. 8 Average energy consumption versus number of biosensors

drop and a high delay rate. Also, it lacks support for mechanisms that accommodates any emergency data [13].

4.2.4.5 Priority-guaranteed MAC protocol Note, a superframe structure performs a major role in the development of a MAC protocol. As a consequence, the priority-guaranteed protocol employed a new superframe structure. Here, the time-axis is composed of the inactive and the active phases. The active phase is divided into five sections in order to accommodate different types of data flow that include a control-channel AC1, a control-channel AC2, a beacon, a time slot reserved for bursty traffic (TSRB), and a time slot for periodic traffic (TSRP). The AC1 as well as the AC2 are used for the UL control of processes, for instance, the control of life-threating medical applications by AC1, and the consumer electronics by $\mathrm{AC} 2$. It is important to emphasize that the $\mathrm{AC} 1$ and $\mathrm{AC} 2$ uses the randomized ALOHA for the TSRB and TSRP processes as shown in Fig. 9 [54].

The TSRB and TSRP are the two reserved time slots for bursty and periodic data respectively. Beacon is used for the biosensor devices synchronization. In addition, the priority-guaranteed protocol is a TDMA based scheme which assigns the GTS to the biosensor devices for the communication of data within the TSRB and TSRP.

The priority-guaranteed MAC in [54] was proposed to meet different service requirements such as energy efficiency, network reliability, and low latency access for medical applications as well as cater for the high data rate service requirement for the non-medical (consumer electronics) applications in a WBAN system. The performance of the protocol was studied by comparing it with the IEEE 802.15.4 protocol in terms of energy efficiency. Simulation parameters such as a physical data rate of $250 \mathrm{kbps}$, a frame length of $61.44 \mathrm{~ms}$, and a traffic arrival rate $(\lambda)$ of 1 and 20 were assumed. For a detailed comparison, two performance measures such as the average energy consumption per kb and the mean node energy consumption were used to evaluate the protocols. The simulation results obtained are shown in Figs. 10 and 11.

From Figs. 10 and 11, we observed that the priority-guaranteed protocol is advantageous in the context of energy savings than the IEEE802.15.4 protocol when compared 
using the same traffic arrival rate. For instance, in Fig. 10, when there are 12 nodes in the system and setting $\lambda=1$ and 20 , we noticed that the average energy consumed per kb is about $0.80 \mu \mathrm{J}$ and $0.39 \mu \mathrm{J}$ respectively when the IEEE 802.15.4 protocol was applied, while that of the priority-guaranteed protocol has an average energy consumption of about $0.76 \mu \mathrm{J}$ and $0.25 \mu \mathrm{J}$, respectively. This implies that the energy consumed by the nodes was saved by approximately $16 \%$ and $50 \%$ under low and heavy traffic load, respectively, using the priority-guaranteed protocol. Also, from Fig. 11, we observed that the priority-guaranteed protocol was able to save up to about $10 \%$ and $20 \%$ energy under low and heavy traffic load respectively due to the exploitation of the data channel and the control channel separation, which helped the data channel to be free from collision occurrence. However, there are some drawbacks that could be attributed to this protocol, for instance, the complexity of its superframe structure and the inability to adapt to emergency traffic.

\section{Summary of the WBAN-specific MAC protocols}

This section compares the WBAN-specific MAC protocols in Table 4 based on some critical requirements of WBAN-enabled IoT technology using qualitative analysis.

\section{Overview of short- and long-range MAC protocols for WBAN-enabled loT technology}

\subsection{Short-range technologies and standards}

The short-range MAC protocols of the short-range standards provide a channel access to various network elements, like the biosensor devices, deployed in a body area for communication purposes at the MAC layer over a short distance. The energy spent by the biosensor devices during the channel access process is a major source of energy consumption apart from the energy consumed by the radio transmitter of a biosensor device. Consequently, insight into different short-range MAC protocols, such as IEEE 802.15.4, IEEE 802.15.6, Bluetooth, Wi-Fi, and ETSI SmartBAN, their underlying energy wastage issues, like idle listening and collisions, and their energy consumption requirement and/or power saving mechanisms, like power control and sleep scheduling, is essential since these factors may impact the performance of a WBAN-enabled IoT technology in terms of energy efficiency, throughput, latency, channel utilization efficiency, and reliability.

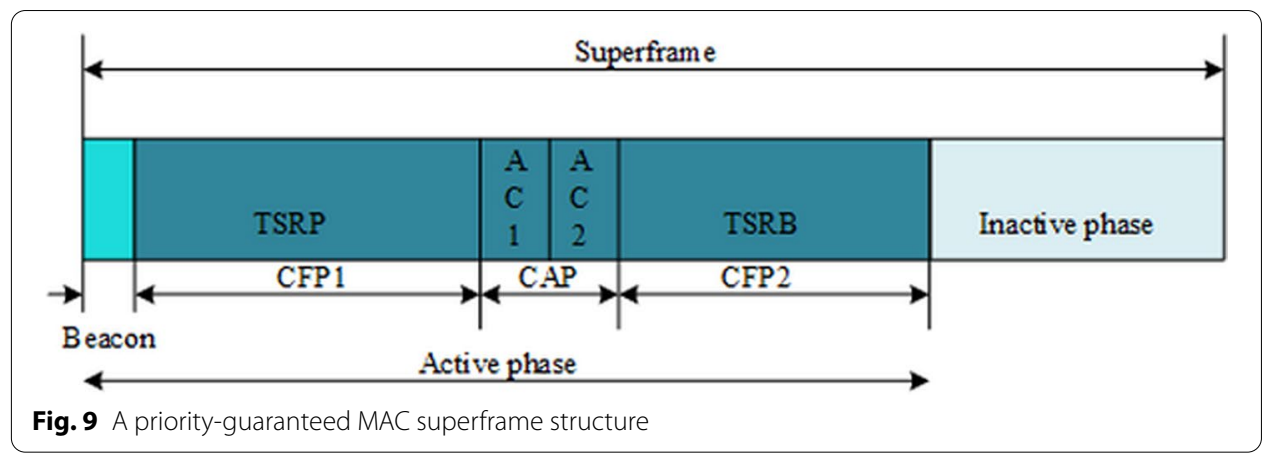




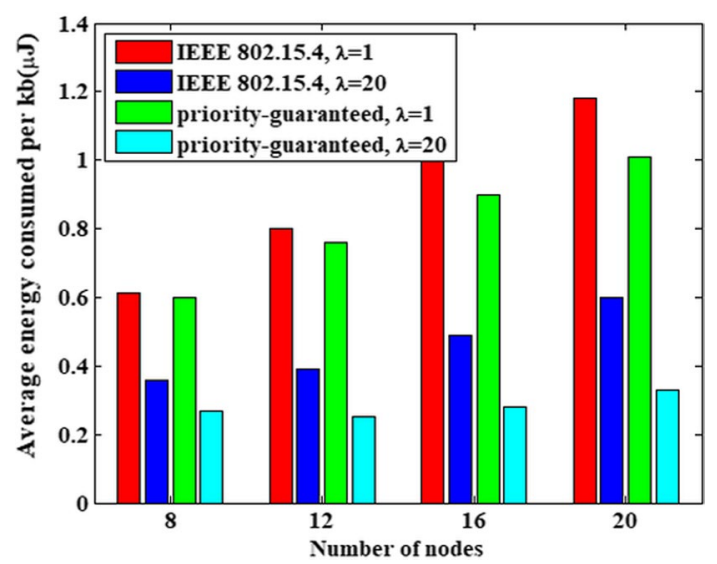

Fig. 10 Average energy consumed versus number of nodes

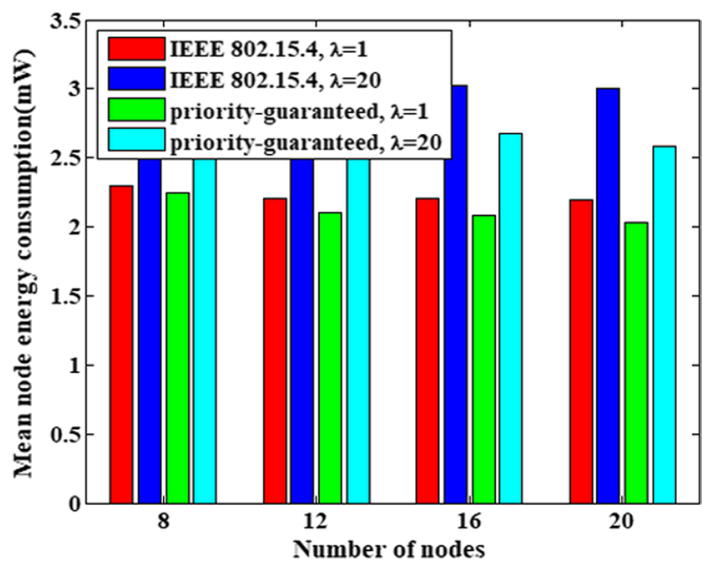

Fig. 11 Mean node energy consumption against the number of nodes

\subsubsection{IEEE 802.15.4 standard}

The IEEE 802.15.4 standard is a complement of the IEEE 802 wireless specifications. It is also described as a low-rate wireless personal area network (LR-WPAN) protocol that operates either on the $2.4 \mathrm{GHz}, 915 \mathrm{MHz}$, or the $868 \mathrm{MHz}$ frequency bands. The main aim of the IEEE 802.15.4 short-range protocol is to provide a low power, low cost, and low packet rate. It also provides support for the wireless connectivity of different types of applications such as medical applications, that include the pervasive and ubiquitous healthcare monitoring systems, home and industrial applications. It is a standard adopted by the ZigBee Alliance and 6LoWPAN for WPAN compliant systems, and defines the PHY and MAC protocol layers for IEEE 802.15.4 systems. The PHY layer defines the network interface parameters, components, as well as their operations. In order to support the MAC layer operations, the PHY layer incorporates several features that can help in energy saving, these include the link quality indicator (LQI), CCA, and receiver energy detection (RED) techniques [55]. Being a short-range standard, an IEEE 802.15.4-enabled biosensor device can achieve about $100 \mathrm{~m}$ communication coverage based on the PHY protocol layer specification and the spectrum employed for 
a geographical area [56]. The MAC protocol layer is responsible for handling network association, disassociation, and controls access to the radio channel that can be realized by two different methods of operation which include the non-beacon and the beacon modes. The non-beacon mode of IEEE 802.15.4 MAC requires the exploitation of the unslotted CSMA/CA technique to perform all transmissions. While, the beacon mode of IEEE 802.15.4 MAC employs the slotted CSMA/CA technique with a superframe structure. $[57,58]$. The superframe structure contains both active and inactive parts. The active part include the CAP, beacon period, and CFP. The inactive part is employed to put the biosensor devices in a sleep state. In the CAP of the active part, the biosensor devices contend for transmission chance on the slotted CSMA/CA protocol. The beacon period is employed to periodically send beacon/control packets by the AP to the biosensor devices for superframe and synchronization definition. The CFP is used by the biosensor devices to request for guarantee time slots (GTSs) for applications that are time-critical. According to [59, 60], the power consumption of the IEEE 802.15.4 standard is typically $\leq 50 \mathrm{~mW}$. The usage of this standard has been explored and applied to WBANs, and a good example is [61]. The authors of [61] considered the extension of the function of the IEEE 802.15.4 standard superframe structure for WBANs to cater for emergency slot and polling access periods. Also, in the proposed solution, a two-hop network was configured to accommodate different kinds of devices putting into consideration the in-body and the on-body communication situations. However, the limitation of this standard could be attributed to its inability to cater for a time-sensitive communication since it operates in the CSMA/CA access mode which waste energy due to issues like idle listening and collisions These issues may make a biosensor device to spend excess energy than what is required for data packets. Another limitation is it inability to provide a full support for the WBAN-enabled IoT QoS requirements [62]. In order to address these limitations, a few number of protocols have recently been proposed in the literature which are not IEEE 802.15.4 based.

\subsubsection{IEEE 802.15.6 standard}

The IEEE 802.15.6 standard describes a MAC layer which provides support for different PHY layers, like the ultra-wideband (UWB), human body communication (HBC), and the narrowband (NB) layers. The UWB is employed to operate in two different frequency bands that include a low and a high band. The low band is made up of three channels

Table 4 Qualitative analysis of WBAN-specific MAC protocols [10, 13]

\begin{tabular}{|c|c|c|c|c|c|c|}
\hline MAC protocols & Energy efficiency & Latency & Throughput & $\begin{array}{l}\text { Channel } \\
\text { utilization } \\
\text { efficiency }\end{array}$ & Reliability & Priority access \\
\hline BodyMAC & Moderate & Moderate & Moderate & High & Low & Low \\
\hline Heartbeat driven & Moderate & High & Moderate & Moderate & Moderate & Low \\
\hline MedMAC & Moderate & High & Low & Moderate & Low & Moderate \\
\hline $\begin{array}{l}\text { Battery-aware } \\
\text { TDMA }\end{array}$ & High & High & Moderate & Moderate & Moderate & Low \\
\hline $\begin{array}{l}\text { Priority-guaran- } \\
\text { teed }\end{array}$ & High & Moderate & Moderate & Moderate & Moderate & Moderate \\
\hline
\end{tabular}


with a mandatory channel of 7 [63]. The NB controls data communication over a network as well as the activation and deactivation of a WBAN radio transceiver, and is also responsible for clearing channel assessment. The HBC make use of the body as a communication medium for transmitting electrical signals, and also utilizes and/or support a frequency band that ranges from 5 to $50 \mathrm{MHz}$. The IEEE 802.15.6 is regarded as the first international WBAN standard [64]. In the IEEE 802.15.6, the biosensor devices may be classified into one hop which support a star topology or two-hop that support a mesh topology [65]. The operation of a WBAN that is controlled by a hub or a single coordinator must have a hub and many biosensor devices that ranges from 0 to $n$ MaxWBANSize, where $n$ MaxWBANSize stands for the maximum WBAN biosensor devices. While, in a two-hop WBAN, a relay-capable biosensor device can be employed to exchange packet frames between a biosensor device and the hub. Additionally, the IEEE 802.15.6 standard separate the channel or time axis into superframes or a beacon period of the same length. Each individual superframe is composed of a number of assigned slots that are exploited for packet transmission.

Moreover, the IEEE 802.15.6 standard employs three communication modes that include a beacon mode with a superframe structure, a non-beacon mode with a superframe structure, and a non-beacon mode without a superframe structure [66]. Also, the IEEE 802.15.6 standard supports three types of access schemes, such as a random access scheme, unscheduled and improvised access scheme (contention-less access), and scheduled-polling access scheme (contention-based and contention-free access) [67]. In the random access scheme, depending on the PHY layer, a hub may exploit either a CSMA/ CA or a slotted ALOHA protocol [68]. The hub consider the CSMA/CA protocol for NB PHY layer and a slotted ALOHA protocol is considered for the UWB PHY layer. In the improvised and the unscheduled access scheme, the hub can employ a make-up access to transfer post or poll command without pre-reservation or a prior notification in the non-beacon with superframe structure or the beacon mode [69]. The scheduled access scheme is exploited to achieve a scheduled bi-link, DL, and UL allocations and a scheduled polling access scheme is utilized for the posted and polled allocation. A comparison and analysis of the IEEE 802.15.6 as well as the IEEE 802.15.4 MAC layer standards were carried out in [70]. Another work is [71] where authors proposed an architecture that uses the IEEE 802.15.6 standard, sensor nodes were attached to the body of different workers in an underground scenario to measure temperature, humidity, gas concentration air flow, and so on. The performance of the system proposed was evaluated in the context of energy consumption, throughput, and delay. Their analysis revealed that the standard is a reliable communication network that can help to achieve a reliable and reasonable quality of service in tunnels and underground environments. However, the standard does not cater for QoS priority architecture which can accommodate all kinds of traffic at the biosensor device side, like the normal and emergency traffic, and at the coordinator side, like the on-demand and normal traffic. Also, since it employs either a CSMA/CA or a slotted ALOHA protocol, energy wastage may be experienced due to potential collisions and idle listening issues. To address these, authors of [72] proposed a transmit energy control mechanism that can adapt to body channel variations, enables constant energy conservation, and prolong the biosensor devices battery lifespan. Note, 
the power transmission requirement of the IEEE 802.15.6 standard is approximately 1 $\mathrm{mW}$ at a distance of $1 \mathrm{~m}[60,73]$.

\subsubsection{Bluetooth/IEEE 802.15.1}

The Bluetooth technology is defined as a WPAN IEEE 802.15.1 standard whose main objective is to allow wireless communications between a number of biosensor devices over a short range. The primary medium access control scheme in the Bluetooth technology is a centralized TDMA based scheme [74]. The Bluetooth technology consists of a baseband layer that carries out the Bluetooth's PHY as well as the Bluetooth's MAC processing [16]. The baseband layer as defined by Bluetooth operates on an unlicensed Industrial Scientific and Medical (ISM) frequency band of $2.4 \mathrm{GHz}$. Additionally, the Bluetooth PHY layer transmission method is based on a frequency hopping spread spectrum (FHSS). Typically, there are 79 radio frequency channels with $1 \mathrm{MHz}$ spacing, however some countries allow the use of 23 channels only.

It is important to mention that the communication in a Bluetooth network is based on a master-to-slave approach where a set of biosensor devices that shares a common communication medium is called a piconet. Each of the piconet is composed of a master biosensor device which helps to control the access to the radio channel and a maximum of seven slaves' biosensor devices. While a group of piconets forms a scatternet [75]. Moreover, gaining access to the radio channel is controlled through the use of a slotted time-division duplex (TDD) technique in such a way that the master biosensor device employs a polling protocol to assign time slots to the slave biosensor devices [76]. The exploitation of this standard in WBAN has been considered in literature, for instance [77], where an investigation on the usage of Bluetooth for an on-body transmission was considered.

For the purpose of energy consumption optimization, the Bluetooth defines four different operational states, including the active state, hold state, sniff state, and park state [24]. The active state allows the master to schedule the health packet transmission based on the traffic demands of different slaves. The sniff state aims to minimize the duty cycle of a slave biosensor device sensing activity. The hold state allows a slave biosensor device to go into a sleep mode for a particular time, i.e., the hold time, and when this time expires, the slave biosensor device can then return to an active state. Likewise, the slave biosensor device remains in the sleep mode for an indefinite time in the park state, while the master biosensor device wakes the slave for it to enter into the active state. The Bluetooth technology has a transmission power that ranges from $-20 \mathrm{dBm}$ to $20 \mathrm{dBm}$, i.e., $0.01 \mathrm{~mW}$ to $100 \mathrm{~mW}$, and a receiver sensitivity of about $-70 \mathrm{dBm}$ to $-82 \mathrm{dBm}$ depending on the physical layer employed [78]. Additionally, the Bluetooth technology was developed to provide a low operational cost, low latency, and a high data rate transmission. Due to the mentioned characteristics, the Bluetooth technology could be employed in a WBAN-enabled IoT technology that requires a high data transmission, but then, this may incur a high energy dissipation cost [79]. Also, since Bluetooth is based on a TDMA scheme, then more energy is required for synchronization. Other limitations of the classic Bluetooth include inadequate standby modes, limited connecting devices, and a slow start-up time. These drawbacks resulted to a new version of the Bluetooth technology which is referred to as the Bluetooth low energy (BLE) or Bluetooth 4.0. The BLE was 
proposed by the Bluetooth Special Interest Group (BSIG) and this group was responsible for the development of the Bluetooth technologies as well as the design of its technical specifications. The BLE technology is mostly employed in devices that only transmits data in a periodic manner and this technically makes it to go to sleep when inactive, that is, when there are no data to transmit. Furthermore, the BLE may achieve energy efficiency by employing low energy chipsets. These chipsets help to realize an ultra-lowpower utilization at different operation modes, including the transmit, receive, as well as the idle modes. It also integrates energy saving mechanism, including adaptive and low duty cycling methods.

\subsubsection{Wireless Fidelity (Wi-Fi)/IEEE 802.11}

The evolution of the IEEE 802.11 standards, i.e., the IEEE $802.11 \mathrm{a} / \mathrm{b} / \mathrm{g} / \mathrm{h} / \mathrm{ah}$ is merged together to form the Wi-Fi technology. This technology was designed to cater for the applications that demand high data transmission rate. Wi-Fi complaint devices could operate on the unlicensed ISM frequency bands of 5 and $2.4 \mathrm{GHz}$ at a high data transmission rate that spans between $11-54 \mathrm{Mbps}$. The high data rate transmission of a Wi-Fi technology could be attributed to its ability to utilize various efficient coding techniques. It also has the ability to cover a communication range of more than $100 \mathrm{~m}$. Note that, this technology is an internet-enabled solution which could be used to provide an internet connectivity in WBANs. Wi-Fi is also considered advantageous over other communication technologies in the short communication range category in terms of high speed and high data transmission rate [80]. It is worth noting that the CSMA/CA protocol is mostly employed as the channel access mechanism in the IEEE 802.11 standard [81]. Similarly, the IEEE 802.11 comprises of two channel access methods that include the distributed coordinator function (DCF) and the point coordinator function (PCF). The DCF is a fully distributed protocol, while the PCF is a centralized protocol [82]. The DCF access the channel through the CSMA protocol with an immediate MAC level acknowledgment and employs an RTS or CTS optional exchange method. To improve the efficiency of this protocol in WBANs context, a research work was carried out in [83] to improve the power saving technique of the DCF IEEE 802.11 in order to enhance its energy efficiency. In the PCF, an AP provides a distributed access, which contains a contention period and contention-free period access, to the channel using a polling method [84]. It is worthy of note that Wi-Fi could either be in a doze mode or wake-up mode. In the doze mode, the biosensor devices cannot transmit or receive any data. The doze mode is technically use for power consumption optimization. Similarly, the Wi-Fi technology can support two major power management mechanisms, including the power save (PS) and active mode (AM) $[85,86]$. However, since the IEEE 802.11 standard uses the CSMA/CA based protocols, then, its ability to fully satisfy the WBAN-enabled IoT QoS requirements remain an issue since the protocol cannot easily scale as the size of the network devices is increased. Another concern is energy wastage due to control overhead packets, idle listening, and collisions. The power consumption of Wi-Fi standard is typically around $800 \mathrm{~mW}[60]$. 
The exploitation of this standard has been considered in a WBAN context, an example is [87]. The authors of [87] proposed a scheme for a mobile Wi-Fi to enable the connectivity of a WBAN system.

\subsubsection{ETSI SmartBAN}

A technical committee was established by the European Telecommunications Standards Institute (ESTI) in March 2013 to propose a smart body area network (SmartBAN) standard defined as the ETSI SmartBAN. This standard could be regarded as a simple, low-power communication, and low complexity system, which enables a wireless connection between biosensor devices and a hub. It exploits a multiple-radio technique to connect biosensor devices by exploiting the existing radio standards, and operates on the frequency band of $2.48 \mathrm{GHz}$. The aim of the SmartBAN is to define an European standard for BAN with focus on some applications, like health care, leisure, sports, wellness, and so on. The main features of this protocol include the ability to access a channel with ease, data transfer reliability, minimal listening period, and ability to provide an additional control message for biosensor devices with low duty cycle [88]. Also, there is a provision for a multi-user channel access (MCA) mode which enables a high priority message transmission, for example, the emergency packets. Furthermore, the MCA helps to achieve a low latency for time-sensitive use cases. On the contrary, the aforementioned features are only specific to SmartBAN, and therefore could not provide support to other WBAN standards, for example, the IEEE 802.15.6 standard. Note, the MAC protocol of the SmartBAN is divided into two access channels, namely the control channel and the data channel. The control channel is used for controlling traffic, the transmission of control messages, and network initialization, while the data channel is used for transmitting data [3]. In addition, the data transmission has an inter-beacon interval which is divided into three major components, namely the inactive period, scheduled access period (SAP), and control and management period (CMP). Note, each period is also divided into different slots of time with the same length. The CMP is based on the slotted ALOHA scheme and is used for data and control transmission. The SAP is majorly for transmitting data and is based on the TDMA scheme which helps to prevent a collision occurrence. An overview of the ESTI SmartBAN MAC protocol was carried out in [89]. As with channel and/or time slot resource pre-allocation protocols, slot wastage and control overhead are potential related issues with this standard.

\subsection{Long-range technologies and standards}

The long-range MAC protocols of the long-range standards allow biosensor devices and base station nodes to access a radio channel for data exchange purposes over a long distance. Since biosensor devices are mostly battery powered devices that could not afford to waste energy on channel access, then, insight into the operation of the long-range MAC protocols of the long-range standards will assist in improving the productivity and performance of WBAN-enabled IoT technology. To achieve this, the low-power wide area network standards are considered in this section. 


\subsubsection{Low-power wide area networks (LPWANs)}

The LPWAN technologies can be classified into two groups, namely the non-cellular LPWAN technologies and cellular LPWAN technologies. The examples of the non-cellular LPWAN technologies are LoRa Alliance, SigFox, and so on. These technologies use proprietary solutions for connectivity, while the cellular LPWAN technologies are new standards, for example, the NB-IoT and eMTC (LTE Cat-M1), and they are regraded as the major foundational building blocks of the $5 \mathrm{G}$ technologies. These new standards are promising since $5 \mathrm{G}$ networks promise to offer high data rate transmission, better reliability, as well as a high bandwidth unlike the classical cellular technologies that was not originally designed for WBAN use cases. The identified standards are considered in the following sub-sections under two categories.

\section{6 (a) Non-cellular LPWAN technologies}

\section{(i) LoRa alliance}

LoRa alliance is based on two main components, including LoRa and LoRaWAN [90]. LoRa is associated with the PHY layer and operates on the unlicensed ISM band and also employ a chirp spread spectrum (CSS) modulation scheme with a data rate of about $300 \mathrm{bps}-50 \mathrm{kbps}$. LoRaWAN on the other hand, is related with MAC layer and uses the pure ALOHA protocol to enable a UL communication in a star topology [4]. It is important to point out that LoRaWAN is designed majorly for low energy consumption devices, like biosensors. Also, the LoRaWAN protocol categorizes LoRa biosensor devices in the context of a WBAN-enabled IoT into three major operation modes which enables a trade-off between the energy requirement, latency, and DL communication. These operation modes include the class A, B, and C. Basically, a LoRa-enabled biosensor device is mostly designed as a class A device with a power saving capability, except in some cases in which it could be specifically designed to function in the Class B or the Class $\mathrm{C}$ mode. The Class $\mathrm{A}$ is regarded as being the most energy efficient class, with a high latency, and offers a DL communication only for a small slot after a UL communication. It has two receive windows. Hence this class may be considered suitable for the WBAN-enabled IoT technology since the DL would only need to acknowledge the delivery of the health data, except that latency will be an issue. Class B support biosensor devices that have a moderate latency requirement and consumes less power with multiple scheduled DL slots. While, Class C supports devices with the lowest latency requirement with continuous open receive windows at the expense of more power cost compare to the Class A and B $[91,92]$. Typically, the energy consumption of this standard is around $\leq 50 \mathrm{~mA}$ for data transmission, $10 \mathrm{~mA}$ to $\leq 40 \mathrm{~mA}$ for reception, and $\leq 0.01 \mathrm{~mA}$ for the sleep mode [3]. However, since the LoRaWAN protocol defines the pure ALOHA scheme as the channel access protocol for classes A, B, and C, then, the collision problem remains a major constraint. Despite the different operation modes available, collisions are still inevitable. Another serious concern is idle listening.

\section{(ii) SIGFOX}

The Sigfox standard employs an ultra-narrow band (UNB) technology and supports a frequency band of $868 \mathrm{MHz}$ in Europe and $915 \mathrm{MHz}$ in the US and a maximum 
throughput of about $100 \mathrm{bps}$ resulting into an ultra-low-power consumption and a low noise level. At the MAC layer, the UNB technology is based on the ALOHA protocol medium access (MA) technique known as the random frequency and TDMA (RFTDMA) protocol. This protocol enables an active biosensor device to randomly access the channel in frequency and time and has no MA collision control approach as well as no prior awareness of the channel occupancy [93]. The RFTDMA protocol helps to achieve a low energy consumption due to the fact that the biosensor devices do not have to sense the channel before transmitting their data. Additionally, the frequencies used for transmission are randomly chosen in a continuous interval. On the contrary, the limitation of this protocol is the chances of collision occurrence which could be attributed to the simultaneous packet transmissions. The UL transmission power of the Sigfox technology depends on global regions which varies from around $14 \mathrm{dBm}$ to $22 \mathrm{dBm}(25 \mathrm{~mW}-158$ $\mathrm{mW}$ ), while the DL transmission power varies from around $26 \mathrm{dBm}$ to $36 \mathrm{dBm}$, i.e., 500 $\mathrm{mW}-4 \mathrm{~W}$, in Europe and United states, respectively. As a consequence, the Sigfox technology uses two power saving mechanisms, including the power saving mode (PSM) and discontinuous reception (DRX) [94]. It is important to point out that in a Sigfox system, a Sigfox-enabled biosensor device does not need to be awake at a particular instance for synchronization, i.e., it is an asynchronous technology. Also, biosensor devices are in the transmission mode when communicating their data and in the sleep mode in between transmissions. Furthermore, the Sigfox technology uses an inter-transmission period in which a biosensor device could be in an idle state [92], due to this idle state, energy may be wasted.

\subsection{Cellular LPWAN 5G IoT radio technologies}

One of the major limitations of the existing communication standards often employed in WBAN-enabled IoT healthcare systems is the limited bandwidth constraint required to efficiently monitor patients' health conditions and report sensory data in a real-time fashion. To address this limitation, the new 5G standards are promising candidates to improve the bandwidth limitation confronted by the WBAN-enabled IoT technology. While most of the existing technology MAC protocols are leveraged on some adaptations to meet some of the various requirements of the WBAN-enabled IoT services, like wide communication coverage, energy efficiency, cost-effectiveness, low delay rate, timely and reliable packet delivery. Fortunately, the 5G technology MAC protocols are envisaged to meet the WBAN-enabled IoT requirements. Additionally, the 5G technologies are also envisioned to provide high data rates, high bandwidth, and also introduce a transport network structure in their architecture so as to offer a sustainable packet communication through the use of a self-backhauling mechanism [95]. Note that the 5G communication systems have self-organizing capabilities that may be exploited through the selection of suitable and available spectrum blocks for backhauling purposes, and this would be an essential feature for offering a high spectrum frequency radio access. Also, according to [96], the 5G communication systems are supported by several medium access mechanisms. The multiple medium access mechanisms include the non-orthogonal multiple access (NOMA), orthogonal frequency-division multiplexing (OFDM), sparse code multiple access (SCMA), coded access reservation, coded slotted 
ALOHA, filter bank multicarrier offset QAM (FBMA-OQAM), interleave division multiple access (IDMA), coded random access, and universal filter OFDM (UF-OFDM). For insight into 5G technologies, the fundamental technologies are discussed in the next sub-sections.

\section{(i) Enhanced machine-type communication (eMTC)}

The enhanced machine-type communication (eMTC) technology is also known as the long term evolution category M1, i.e., LTE Cat-M1 technology. The eMTC technology was developed to provide packet reliability, device identification, low complexity, and security [97]. The eMTC was designed according to the 3GPP specifications so as to realize a lowpower consumption as well as extend the existing communication technology coverage. The 3GPP design aim for the eMTC communication technology was to provide suitable MAC and PHY connections for the eMTC networks as well as optimize the communication access technology, support flexible bandwidth deployment, increase spectral efficiency, reduce delay, support high throughput rate, and reduce idle time [98]. The eMTC networks are regarded as a multicarrier network that spreads radio resources in the frequency domain and in the time domain by employing a frequency-division duplex (FDD) method and a single carrier (SC-FDMA) mechanism as well as a TDD method and an orthogonal frequency-division multiple access (OFDMA) scheme for UL and DL health packet transmissions, respectively. For the UL health packet transmission, there is only a single physical UL control channel (PUCCH) which conveys information that include the channel quantity information (CQI), the transmission confirmations, and the scheduling requests. Also, a physical random channel (PRACH) method is employed to estimate the arrival period of the UL message as well as to initiate a connection between the base station and the terminal [99]. Note that the eMTC-based biosensor devices can support a multiple input multiple output (MIMO) data communication and thereby allowing the base station to send various data streams concurrently over the same carrier [100].

Typically, the MAC layer exists in the universal equipment (UE) as well as the evolved Node-B (ENB) and is a component of the eMTC air interface control and user planes [101]. The radio access network protocol in eMTC is the evolved universal terrestrial radio access network (E-UTRAN). Basically, the main functions and services of the MAC protocol include multiplexing/de-multiplexing of the service data units (SDUS) of MAC which belongs to a single or several logic channels into or form a transport block (TB) carried to and from the PHY layer on the transport channels, report scheduling information, correct error through hybrid automatic repeat request (HARQ), handling priority between the LCs and the UEs centered on dynamic scheduling and so on [102]. Furthermore, the eMTC MAC protocol employs scheduling mechanisms in order to schedule health packet transmissions so as to guarantee health packet successful transmission in the context of the WBAN-enabled IoT to provide a promising achievable throughput, and a spectrum efficient transmission of the health packets.

Generally speaking, the power wastage of a biosensor-enabled eMTC device could be attributed to the periodic listening of paging signals and measuring of the link quality. While, the energy consumption due to data transmission is insignificant of the total energy consumed. Consequently, the eMTC exploits two power management 
mechanisms for prolonging the battery lifespan of biosensor devices, these mechanisms include the PSM and DRX. In practice, the PSM is used to enable biosensor devices to turn off energy consuming functionalities for the purpose of saving energy. While, the DRX works by providing a support to a biosensor device by reaching an agreement on how to manage data communication during the data reception period with the network, thereby enabling a biosensor device to keep its receiving functionality off to save energy. In addition, the eMTC was developed to support a transmission power of either $23 \mathrm{dBm}$ or $20 \mathrm{dBm}$ [103].

\section{(ii) Narrowband-internet of things (NB-IoT)}

The NB-IoT technology can as well be referred to as the LTE Cat-NB1 and is designed to extensively reuse the architectural design of the LTE networks [104]. This technology may support most of the key requirements for data communication in a WBAN-enabled IoT remote healthcare monitoring system that include low-power dissipation, low complexity, and long-distance communication. The NB-IoT technology employs a single-carrier SC-FDMA scheme for the UL transmission and the OFDMA for the DL transmission. Based on the SC-FDMA scheme, multiple access is made possible to biosensor devices by allocating different sets of overlapping-free subcarriers. Moreover, the NB-IoT may provide two types of transmissions for the health packets communication in the context of WBAN-enabled IoT technology, they include the single-tone and multi-tone transmissions. The multi-tone transmission is centered on the SC-FDMA with $0.5 \mathrm{~ms}$ slot, $15 \mathrm{kHz}$ sub-carrier spacing and $1 \mathrm{~ms}$ subframe as the same as the LTE networks. While, the single-tone transmission makes use of $3.75 \mathrm{kHz}$ with $2 \mathrm{~ms}$ slot period and $15 \mathrm{kHz}$ sub-carriers spacing for UL transmission [105]. Additionally, the UL of the NB-IoT contains two physical mediums, i.e., channels that include the narrowband physical UL shared channel (NPUSCH) and narrowband physical RACH (NPRACH). There are two distinct formats in the NPUSCH channel, the first format is utilized when loading an UL packet and has a peak block size of about 1000 bits [106], and the second format employs a repeat code for correcting errors as well as a signal HARQ for NPUSCH recognition. Just like the eMTC technology, the NB-IoT has a maximum power transmission of about $20 \mathrm{dBm}$ or $23 \mathrm{dBm}$ [103]. However, periodic listening to paging signals and the channel quality measurement functions performed by the NB-IoT-enabled biosensor devices are the major sources of energy consumption of this technology. To optimize the energy consumption of this technology, two power saving mechanisms could be employed by the NB-IoT technology, which are the PSM and the DRX. This standard may suffer from collisions when multiple biosensor devices simultaneously request access to the channel resources because of the likelihood of false alarm, inaccurate channel estimation and channel detection issues related to the NPRACH [107].

\section{Summary of short- and long-range MAC protocols for WBAN-enabled IoT technology}

The summary and the comparison of both the short- and the long-range MAC protocols for WBAN-enabled IoT technology with focus on communication coverage, energy efficiency, data delay rate, transmission rate, power consumption, access methods, advantages, and limitations are provided in Table 5. 


\section{Discussion on open-ended research problems and future directions for WBAN-enabled loT technology's MAC protocols}

This section presents key discussions on the open-ended research problems associated with the WBAN-enabled IoT technology's MAC protocols as well as future directions for improving the productivity of these systems. The design of energy efficient MAC protocols is a pragmatic idea for improving the network efficiency of WBAN-enabled IoT technology. Currently, the design of MAC protocols is an active research area in the domain of WBANs as the existing ones have not fully catered for the efficiency requirements of WBAN systems, while some are presently at their infancy stage. As an example, the new communication technologies are still in need of new novel MAC protocols that can be suitably adapted into the WBAN-enabled IoT technology for enhancing their efficiency, for instance energy utilization optimization and delay optimization. As a consequence, this study has put forward some recommendations for future research directions that can help to improve the efficiency of the MAC protocols that target WBAN-enabled IoT technology.

(1) Exploring and exploiting hybrid methods for WBAN-enabled IoT MAC protocols

The hybrid method is an interesting concept that can be employed to combine the strengths of different MAC protocols in the categories of contention-free and contention-based MAC protocols to improve the QoS performance of WBAN-enabled IoT. But then, exploiting this concept often results into performance trade-off issues that requires novel ideas to increase the performance efficiency of hybrid MAC protocols. For example, the combination of both TDMA and slotted ALOHA MAC protocols is a potential hybrid MAC protocol. However, when the TDMA and slotted ALOHA are integrated into a WBAN-enabled IoT to provide channel access to the biosensor devices, where the TDMA protocol is employed as a transmission mechanism and the slotted ALOHA protocol is employed as a contention mechanism, there will be likelihood of a collision occurrence when two biosensors transmit their packets at the same time-slot during the contention period as well as performance trade-off between the contention time and the transmission time. Addressing these issues are key to developing efficient hybrid MAC protocols for WBAN-enabled IoT. To address these issues, interesting future research directions include the design of modified slotted ALOHA protocol to minimize collisions by employing techniques from graph and tree theories and balancing the tradeoff issue between the contention time and the transmission time using techniques from operations research.

(2) Addressing the channel access issues of LoRaWAN MAC in WBAN-enabled IoT

LoRaWAN is a potential communication protocol that can be integrated into the WBAN-enabled IoT technology to improve QoS performance, however, there is a need to address the channel access issues related to the LoRaWAN MAC. The LoRaWAN MAC is based on the ALOHA channel access scheme and provides a channel access to the network devices in a random manner [108]. Consequently, the LoRaWAN MAC enables devices to randomly select an uplink channel to send their data without the consideration of any other transmission, leading to collisions between several transmissions 


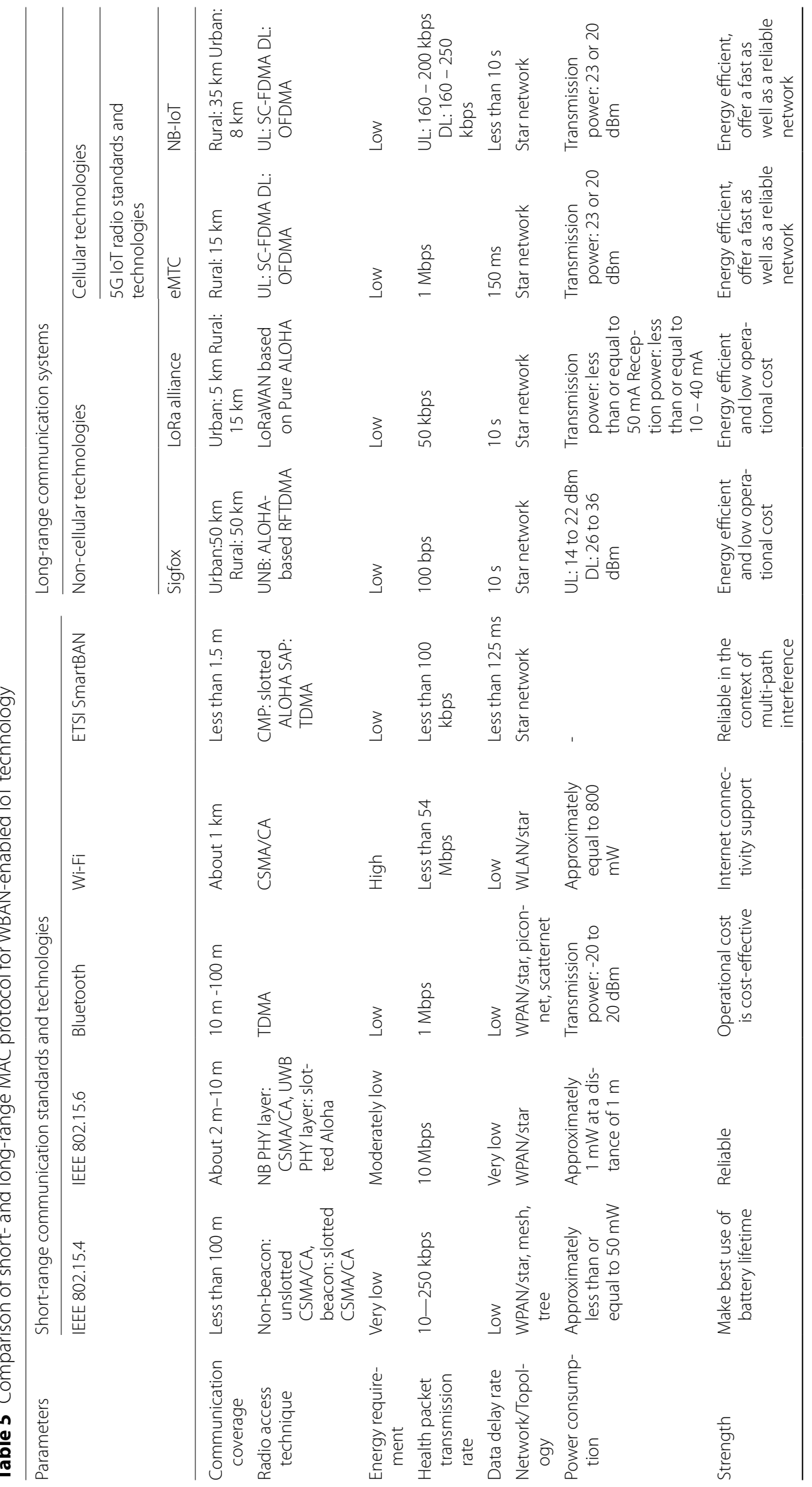




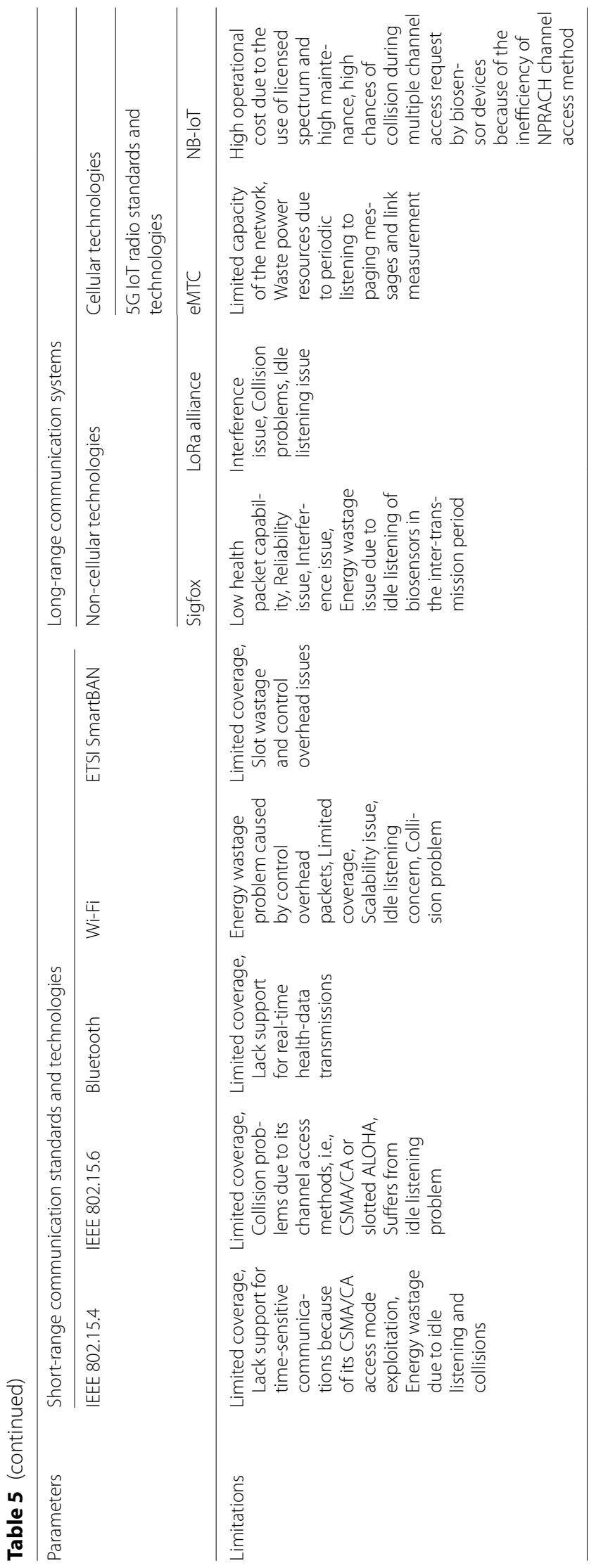


due to simultaneous channel utilization. This collision issue is envisaged to be an underlying problem in a WBAN-enabled IoT in terms of QoS performance, energy efficiency and delays for example. For the LoRaWAN technology to be successfully integrated into the WBAN-enabled IoT for better QoS performance, further research is needed to be conducted on this.

(3) Addressing the NB-IoT technology channel access issues in WBAN-enabled IoT

NB-IoT is a promising technology that can be combined with WBAN-enabled IoT to improve QoS performance, but then, the channel access mode of this technology causes a serious performance issue that calls for more research intervention. For example, when multiple devices request for the uplink channel resources simultaneously to send their data, there may be collisions between several transmitting devices due to various factors like inaccurate channel estimation, inefficient channel detection, and false alarm, and the consequences of the collisions are retransmissions and power wastage issue. Currently, the collisions issue still remain a serious limitation to the deployment of NB-IoT in WBAN-enabled IoT. Hence, there is a need for more investigations on the design of new techniques on efficient channel estimation and accurate channel detection to deal with the collisions issue and to advance the NBIoT for WBAN-enabled IoT.

(4) Supporting heterogeneous and variable health data traffic in WBAN-enabled IoT

Because of the various heterogeneous biosensor devices and varying delay and throughput requirements associated with the WBAN-enabled IoT, such a technology is required to take into consideration and support heterogeneous traffic and variable traffic. For this purpose, research on the design and development of heterogeneous traffic-aware and variable traffic-aware MAC for WBAN-enabled IoT technology is very critical to dynamically allocate channel resources in response to the health data traffic.

(5) Development of optimized clustering techniques for WBAN-enabled IoT MAC protocols

Battery power conservation, increasing network lifetime, and scalability are important factors to be taken into account when designing MAC protocols for WBAN-enabled IoT to make them sustainable and support more biosensor devices. To address this, the exploitation of clustering technique is a promising future research direction to reduce the power path cost of the biosensor devices when communicating with the AP. For examples, the use of clustering technique makes it possible to group biosensor devices into several clusters with cluster heads and the biosensor sensor devices in each cluster communicates directly with their respective cluster heads instead of expending more power on communicating directly with the AP. Also, the use of clustering technique can help to achieve a more scalable MAC protocol for WBAN-enabled IoT.

(6) Developing efficient wake-up-enabled MAC protocols for WBAN-enabled IoT

Because of the significant amount of power consumed by the biosensor devices during the active state, it will be reasonable to combine a passive wake-up radio with the 
main radio in the biosensor devices to allow the main radio to be in a sleep state and to circumvent the power wastage issue due to the unnecessary idle listening of the main radio. In this case, the wake-up radios are enabled to monitor the environment and to listen to the request from the AP $[109,110]$. The usage of passive wake-up radios are useful to increase energy efficiency and to reduce collisions by switching on only the biosensor devices that need to either receive or transmit packets at a wake-up tone message request from the global wake-up radio in the AP. This wake-up tone signals are coordinated by the MAC protocol and are broadcasted on a different channel other than the one used by the biosensor devices for health data communication purposes to avoid collisions between the wake-up tone messages and health data packets. The use of a passive wake-up radio will be advantageous in WBAN-enabled IoT settings since it is a low-cost device and harvest its power from the incoming wake-up tone signal. Also, it requires a very low power compared to the conventional duty cycling techniques A passive wake-up radio can use about $50 \mu \mathrm{W}$ energy and this makes it a promising candidate for the WBAN-enabled IoT technology [30, 109]. However, the current MAC protocols employed to wake up the biosensor devices from a sleep mode are still not efficient and could be further improved on to reduce the delays associated with harvesting power and transitioning from the sleep mode to the active mode when sending critical health data in emergency cases.

(7) Developing efficient time-based MAC protocols for WBAN-enabled IoT technology

As a result of the power wastage issues related to the existing MAC protocols designed for WBANs because of collisions, idle listening, and overhearing issues as well as the transitions that occurs between the different operation modes (i.e., active, sleep, idle), the usage of the channel resources by the biosensor devices may be efficiently timed by researching, designing, and developing novel time-based channel access MAC protocols that are suitable for deployment in WBAN-enabled IoT. Also, the investigation of a time-based channel access MAC protocol can improve delay prediction and minimize health packet drops because of buffer overflow or interference constraints. However, addressing the above-highlighted issues through the researching of time-based channel access MAC protocols may increase the network overheads. To deal with the likely incurred overheads, another focus of research is to consider the idea of a reservation-based request along with simplified heuristic techniques to handle the complexity of the channel access scheduling.

(8) Design of priority access MAC protocols for WBAN-enabled IoT critical data

Due to the varying traffic load or the characteristics of the biosensor devices in the WBAN-enabled IoT technology, there are still much room for the extension of the current MAC protocols so as to provide an improved and efficient priority support for the WBAN-enabled IoT applications, especially the medical applications with time critical data and other requirements. Such priorities could be classified into normal data, periodic data, and critical data, i.e., emergency data and data delay requirement. As an example, some WBAN-enabled IoT applications are delay intolerant, while some are delay tolerant such as the non-medical applications. Since life is involved, critical health data with stringent data delay requirements, typically needs to be 
reported as soon as possible to the designated remote healthcare providers in less than a second, then, the provision of prompt access to the communication medium for such critical health data must be highly prioritized [111]. Unlike the other health data which are less critical. In addition, the energy resource can be efficiently used by introducing a priority differentiation approach. For this reason, by utilizing a random function, the radio channel access may be controlled with the help of allocating priorities to the biosensor devices or the channel links to the destinations. While, the biosensor devices with the highest priority may be allowed to access the channel first.

In the area of priority access MAC protocol, some efforts have been intensified, for example [112, 113], while at the same time some short-range standards, like the IEEE 802.15.4, IEEE 802.15.6, and ETSI SmartBAN, may provide priority access and/ or high-priority messaging support, however, since the WBAN-enabled IoT technology will potentially rely on the IoT technologies that have capabilities for transmitting a few number of data packets because of their low-power features, the investigation of energy efficient and priority access aware MAC protocols deserve more research attention and is currently regarded as an active area of research.

(9) Investigation of new low duty cycling techniques for WBAN-enabled IoT MAC protocols

The duty cycling technique is an energy saving scheme [17] that may be integrated into the WBAN-enabled IoT MAC protocols for optimizing the way the biosensor devices access the radio channel. The basic concept of a low duty cycle technique is to minimize the time a biosensor device spends on idle listening or overhearing needless activities going on in the radio channel by putting the biosensor device in a sleep mode [114]. Duty cycle can be determined by the ratio of the idle listening time length to the wakeup time length and this helps to determine the duration a biosensor device spends in the idle listening period. A duty cycling protocol can be grouped into two categories that include the fixed duty cycle and the adaptive duty cycle. An example of a protocol that employs the fixed duty cycle idea, i.e., they make use of fixed length of time, is the sensor-MAC (S-MAC) protocol. While, the examples of the adaptive duty cycle protocols are the wireless sensor MAC (WiseMAC) and the T-MAC, which adaptively determines the sleep and the wake time of a biosensor device. Typically, the adaptive duty cycling techniques are considered to be more effective in conserving energy than the fixed duty cycling technique $[115,116]$. Other examples of the duty cycle MAC protocols include the energy adaptive clustering hierarchy, power aware cluster TDMA (PACT), and ALOHA with preamble sampling [117]. However, the need to improve the energy efficiency and latency QoS performance of the existing techniques is still an active area of research in the WBAN research domain.

(10) Development of traffic and topology adaptive MAC protocols for WBANenabled IoT

To improve the energy efficiency of a WBAN-enabled IoT technology, MAC protocols can be optimized to adjust to the traffic condition changes and the network topology changes. But then, adaptability to changes typically could result to an increase in the computational complexity of a protocol including other disadvantages that may make the biosensor devices to expend more energy resources. In the area of traffic adaptive MAC protocol development, some efforts have been intensified in [118, 119], 
however, further investigations are required because of the unique challenges posed by the WABN-enabled IoT technology. Thus, the development of traffic and topology adaptive MAC protocols that captures the effect of topology and traffic changes is still an active research issue that requires more attention for improved energy efficiency in the WBAN-enabled IoT technology.

(11) Developing MAC protocols that support fading and mobility management for WBAN-enabled IoT technology

Because of the mobile characteristics of the WBAN-enabled IoT biomedical devices and the dynamic nature of the environment of this technology, there is a need to take the issue of mobility management into consideration as this affects the total performance of a WBAN-enabled IoT technology because of the shadowing, diffraction, and the reflection of body effect in a typical fading wireless channel [120]. To address this open research problem, an interesting future research direction will be to research, design and develop novel WBAN-enabled IoT MAC protocols that takes fading and mobility management into consideration to guarantee the reliability QoS performance of this technology.

(12) Investigation of QoS support-based MAC protocols for WBAN-enabled IoT technology

WBAN-enabled IoT health data could be classified into ordinary health data, critical health data, delay in-tolerant health data, and reliability sensitive health data. As a result, these health data types require a guaranteed and dedicated QoS so as to transmit their health data with little or no delay or packet loss. Therefore, there is still much room for researchers to explore and develop efficient QoS sensitive MAC schemes that are aware of throughput, delay, path loss requirements, and so on. Also, since path loss issue in the WBAN-enabled IoT could be as a result of patient's different postural movement including swimming, walking, standing, lying-down and so on, hence further research on improving the existing routing and MAC protocols need to be intensified so as to further address the problems that are associated with the path loss issue.

\section{(13) Design of low-complexity MAC protocols for WBAN-enabled IoT}

Typically, the centralized and the distributed access schemes are used in centralized and distributed WBAN networks respectively, for resource allocation purposes such as channel access. For the realization of this purpose, a central AP and the biosensor devices employs control signals for the exchange of useful information in the centralized networks, while the exchange of control signals exists between the distributed APs and the biosensor devices in the distributed networks. Note, the signaling overhead involved in the centralized networks often requires a huge amount of energy as the biosensor expends more energy for exchanging control signals compared to the distributed networks. Unfortunately, the biosensor devices in the WBAN-enabled IoT technology are low-power in nature and could not afford to spend energy unreasonably. To deal with the potential huge burden of the centralized networks on the biosensor devices, the investigation of low-complexity distributed MAC algorithms are active open research problems to maximize the network resources as well as improving the network utility gains. In addition, most of the existing MAC protocols are computationally expensive, therefore, further research on modifying and advancing the current versions of these 
protocols is very crucial to obtain more suitable protocols for the WBAN-enabled IoT technology.

(14) Improving WBAN-enabled IoT technology spectrum/channel efficiency and access strategies

As new wireless communication system paradigms would technically require higher spectrum usage as well as the ability to handle some data hungry devices, and since the conventional spectrum allocation strategies causes spectrum scarcity, hence, new strategies and protocols need to be designed so as to achieve an efficient spectrum utilization [6]. It is important to emphasize that MAC protocols are responsible for identifying available spectrum, scheduling of network resources such as time slots, and coordination of heterogeneous systems as well as users' coexistence [4]. In addition, WBANenabled IoT technology are often confronted with issues like delay, energy inefficiency, and spectrum scarcity problems since they are mostly deployed on the unlicensed overcrowded spectrum which are also used by other wireless networks since it is easily and freely accessible. In order to circumvent most of these issues, an appealing solution is to adopt a cognitive radio (CR) technology $[121,122]$. However, one problem with a CR based WBAN-enabled IoT technology is that the biosensor devices can only access the spectrum to communicate their health data in an opportunistic manner to avoid causing interference to the signals of the licensed primary users. To deal with this problem in the context of WBAN-enabled IoT, considering the time-critical nature of its health data, efficient CR-based MAC protocols are needed to be investigated.

(15) Design of efficient retransmission policies and error correction schemes for WBAN-enabled IoT technology

Based on the review carried out on hybrid MAC protocols in this study, it is apparent that most protocols in this category exploits the CSMA/CA scheme making collision occurrences inherent as a result of frequent data retransmission which could lead to energy wastage. To address this issue, i.e., the frequent data retransmissions, an error correction technique could be employed and this could help to achieve a better energy utilization process. Similarly, another approach to tackle the frequent data retransmission problem is by minimizing collisions through changing the contention window ranges, by applying an additive increase and multiplicative algorithm and by modifying the superframe periods in line with the application requirements. For instance, authors of $[123,124]$ proposed the use of a hybrid automatic repeat request policy to address retransmission issue. As a consequence, further research could still be conducted on designing a cooperative and energy efficient retransmission policy.

\section{(16) Addressing co-existence issue in WBAN-enabled IoT technology}

A WBAN-enabled IoT technology may encounter a co-existence issue since it co-exist together with other general wireless communication systems on the same frequency bands in the unlicensed overcrowded spectrum [125]. The co-existence issue may potentially subject the WBAN-enabled IoT technology to critical interference problems that could degrade its performances in terms of delay due to collisions as well as energy wastage due to retransmissions. Hence, interference mitigation or avoidance access protocols that are interference aware needs to be investigated, developed and incorporated into the WBAN-enabled IoT technology. In this matter, MAC protocols should take into consideration the collaborative and cooperative co-existence with other various WBAN 
systems and the general wireless communication systems. In addition, ideas like time and frequency spacing, standards modification, and code diversity could be further investigated to mitigate interference issues in WBAN-enabled IoT.

(17) Enhancing the authentication security of WBAN-enabled IoT technology via the exploitation of new authentication schemes

IoT authentication security is a key requirement of WBAN-enabled IoT technology due to the possibility of security vulnerabilities (e.g., authentication vulnerability) from attacks [126-129]. Such a security measure helps to guard against any unauthorized disclosure of a patient's health data to the attackers [130]. However, the conventional cryptographic security mechanisms like AES, ECC, and RSA, including other new authentication mechanisms employed for authentication security are not efficient for WBAN-enabled IoT technology, biosensor devices, and users owing to the fact that such security mechanisms are resource demanding [8], and the biosensor devices in healthcare applications are resource-limited devices, hence, these devices may be unable to afford significant resources, including power and memory for security authentication purposes $[126,127]$. To this end, there is a need to investigate and develop efficient and lightweight authentication schemes for achieving IoT authentication security in WBANenabled IoT technology.

\section{Conclusion}

To improve the quality of life as well as achieve the future visions of diagnostics, timely detection, treatment, and prevention of diseases through advanced technologies, wireless body area networks (WBANs) fused with the internet of things technologies (IoT) are powerful and promising solutions. But then, these technologies have posed several unique challenges due to different resource scarcity issues related to communication networks. Also, to efficiently drive these technologies, there must be efficient network support in place, especially at the medium access control (MAC) layer. Because of these requirements, this paper has considered the analysis of WBAN-enabled IoT technology MAC protocol requirements and major issues related to the MAC layer, investigation of the potential MAC strategies that are applicable to WBAN-enabled IoT technology for improving communication quality and supporting critical QoS requirements, summary of the existing WBAN-specific MAC protocols, communication standards for WBAN and their underlying constraints. Based on these, future directions and open research issues have been pointed out to improve the productivity and wide deployment of these technologies.

\footnotetext{
Abbreviations

AM: active mode; AP: access point; BLE: bluetooth low energy; BS: base station; BSIG: bluetooth special interest group; CA: collision avoidance; CA-MAC: context-aware medium access protocol; CAP: contention access phase; CCA: clear channel assessment; CDMA: code-division multiple access; CFP: contention-free phase; CSMA: CS: Carrier sensing multiple access; CTS: clear to send; DL: downlink; ECG: electrocardiogram; EEG: electroencephalogram; EMG: electromyography; ESTI: European Telecommunications Standards Institute; FDMA: frequency-division multiple access; FEC: forward error correction; GTS: guaranteed time slot; loT: internet of things; LR-WPAN: low-rate wireless personal area network; LQI: link quality indicator; MAC: medium access control; MedMAC: Medical Medium Access Protocol; PS: power save; QoS: quality of service; RED: receiver energy detection; RTS: request to send; SmartBAN: smart body area network; TDMA: time-division multiple access; $T_{s}$ : dedicated slot time; TSRB: time slot reserved for bursty traffic; TSRP: time slot for periodic traffic; UL: uplink; UWB: ultra-wideband; WBAN: wireless body area network; WSNs: wireless sensor networks.
} 


\section{Authors' contributions}

All the authors have equally contributed to the review of this article. Conceptualization, D.D.O. and A.A.; resources, D.D.O., A.A. and G.H.; investigation and writing-original draft preparation, D.D.O. and A.A.; writing-review and editing, D.D.O., A.A., and G.H.; visualization, D.D.O., A.A. and G.H.; supervision, A.A. and G.H.; project administration, D.D.O. and A.A.; fund acquisition, A.A. All authors read and approved the final manuscript.

\section{Funding}

This research was supported by the Council for Scientific and Industrial Research, Pretoria, South Africa, through the Smart Networks collaboration initiative and loT-Factory Program (Funded by the Department of Science and Innovation (DSI), South Africa). The APC was funded by the CSIR.

\section{Availability of data and materials}

The authors declare that the data and materials in this manuscript are available.

\section{Competing interests}

The authors declare no competing of interest.

\section{Author details}

${ }^{1}$ Department of Electrical, Electronic and Computer Engineering, University of Pretoria, Pretoria 0001, South Africa.

${ }^{2}$ Council for Scientific and Industrial Research (CSIR), Pretoria 0184, South Africa. ${ }^{3}$ Department of Computer Science, City University of Hong Kong, Kowloon Tong, Hong Kong.

Received: 6 July 2020 Accepted: 4 February 2021

Published online: 18 March 2021

\section{References}

1. D.D. Olatinwo, A. Abu-Mahfouz, G.P. Hancke, A hybrid multi-class MAC protocol for loT-enabled WBAN systems. IEEE Sensors J. (2020). https://doi.org/10.1109/JSEN.2020.3037788

2. D.D. Olatinwo, A. Abu-Mahfouz, and G.P. Hancke, A survey on LPWAN technologies in WBAN for remote health-care monitoring. Sensors, pp.1-26, Nov. 2019.

3. M.M. Alam, H. Malik, M.I. Khan, T. Pardy, A. Kuusik, Y. Le Moullec, T.J. Seebeck, A survey on roles of communication technologies in IoT-based personalized healthcare applications. IEEE Access 6, 36611-36631 (2018)

4. X. Yang, L. Wang, Z. Zhang, Wireless body area networks MAC protocol for energy efficiency and extending lifetime. IEEE Sensors Lett. 2(1), 7500404 (Mar.)

5. N. Aslam, K. Xia, M. Haider, M. Hadi, Energy-aware adaptive weighted grid clustering algorithm for renewable wireless sensor networks. Future Int. 9(4), 1-23 (2017)

6. L. Jing, L. Ming, Y. Bin, L. Wenlong, A novel energy efficient MAC protocol for wireless body area network. China Commun. 12(2), 11-20 (2015)

7. A. Saboor, R. Ahmad, W. Ahmed, A.K. Kiani, Y. Le Moullec, M.M. Alam, On research challenges in hybrid medium-access control protocols for IEEE 802.15.6 WBANs. IEEE Sens. J. 19(19), 8543-8555 (2018)

8. B. Liu, Z. Yan, C.W. Chen, MAC protocol in wireless body area networks e-health: challenges and a contextaware design. IEEE Wirel. Commun. 20(4), 64-72 (Aug.)

9. R. Sruthi, Medium access control protocols for wireless body area networks: a survey. Procedia Technol. 25 , $621-628$ (Jan.)

10. A. Rahim, N. Javaid, M. Aslam, M, Z. Rahman, U. Qasim, and Z. A. Khan, A comprehensive survey of MAC protocols for wireless body area networks. in Proc. IEEE 7th 2012 International Conf. Broadband, Wireless Computing, Communication and Application, Victoria, BC, Canada, Nov. 2012

11. A. S Gopalan and J. T. Park, Energy-efficient MAC protocols for wireless body area networks: Survey. in Proc. IEEE 2010 International Conf. Ultra-Modern Telecommunications and Control Systems, Moscow, Russia, Oct. 2010, pp. 739-744

12. F. Ullah, A.H. Abdullah, O. Kaiwartya, S. Kumar, M.M. Arshad, Medium access control (MAC) for wireless body area network (WBAN): superframe structure, multiple access technique, taxonomy, and challenges. Hum. Centric Comput. Inf. Sci. 7(1), 34 (2017)

13. N. Javaid, S. Hayat, M. Shakir, M. A. Khan, S. H. Bouk, and Z. A. Khan, Energy efficient MAC protocols in wireless body area sensor networks- a survey. arXiv preprint https://arxiv.org/abs/1303.2072. 2018.

14. T. Salman, and R. Jain, A survey of protocols and standards for internet of things. arXiv preprint https://arxiv .org/abs/1903.11549, 2019.

15. J. Kabara, M. Calle, MAC protocols used by wireless sensor networks and a general method of performance evaluation. Int. J. Distrib. Sens. Netw. 8(1), 1-11 (Jan.)

16. L. Oliveira, J.J. Rodrigues, S.A. Kozlov, R.A. Rabelo, V.H. Albuquerque, MAC layer protocols for internet of things: a survey. Future Internet 11(1), 16 (2019)

17. D.A. Hammood, H.A. Rahim, R.B. Ahmad, A. Alkhayyat, M.E.M. Salleh, M. Abdulmalek, M. Jusoh, Q.H. Abbasi, Enhancement of the duty cycle cooperative medium access control for wireless body area networks. IEEE Access 7, 3348-3359 (Jan.)

18. D.A. Hammood, H.A. Rahim, A. Alkhayyat, R.B. Ahmad, Q.H. Abbasi, Reliable emergency data transmission using transmission mode selscetion in wireless body area network. Cogent Eng. 5(1), 198-206 (Jan.)

19. M. Cicioglu, A. Calhan, Dynamic HUB selection process based on specific absorption rate for WBANs. IEEE Sens. J. 19(14), 5718-5722 (Mar.) 
20. W. Saad, S.A. El-Feshawy, M. Shokair, M.I. Dessouky, Optimised approach based on hybrid MAC protocol for M2M networks. IET Netw. 7(6), 393-397 (May)

21. Y.A. Qadri, A. Nauman, Y.B. Zikira, A.V. Vasilakos, S.W. Kim, The future of healthcare internet of things: a survey of emerging technologies. IEEE Commun. Surv. Tutorials 22(2), 1121-1167 (2020)

22. X. Gao, J. Yan, J. Lu, A fairness oriented neighbor-channel-aware MAC protocol for airborne sensor networks. Sensors 17(5), 1-14 (May)

23. S. Ullah, H. Higgins, B. Braem, B. Latre, C. Blondia, I. Moerman, S. Saleem, Z. Rahman, K.S. Kwak, A comprehensive survey of wireless body area networks. J. Med. Syst. (Spinger) 36(3), 1064-1094 (Jun.)

24. K. Sohraby, D. Minoli, T. Znati, Wireless Sensor Networks: Technology, Protocols and Applications (Wiley, New York, 2007).

25. N. Javaid, I. Israr, M. A. Khan, A. Javaid, S. H. Bouk, and Z. A. Khan, Analyzing medium access techniques in wireless body area networks. arXiv preprint https://arxiv.org/abs/1304.1047, 2013.

26. L. Shi, A.O. Fapojuwo, TDMA scheduling with optimized energy efficiency and minimum delay in clustered wireless sensor networks. IEEE Trans. Mobile Comput. 9(7), 927-940 (2010)

27. B. Naveen, V. K. Harsh, and D. Renu, An energy efficient TDMA based MAC protocol for wireless body area networks in Proc. IEEE 2018 1st International Conf. Secure Cyber Computing and Communication, Jalandhar, India, Dec. 2018, pp. 545-549

28. Garg V. Wireless communications \& networking. Elsevier, Jul. 2010, pp.149-191.

29. A. Rajandekar, B. Sikdar, A survey of MAC layer issues and protocols for machine-to-machine communications. IEEE Internet Things J. 2(2), 175-186 (Jan )

30. X. Yuan, C. Li, L. Yang, W. Yue, B. Zhang, S. Ullah, A token-based dynamic scheduled MAC protocol for health monitoring. EURASIP J. Wirel. Commun. Netw. 1, 125 (2016)

31. C. Li, H.B. Li, R. Kohno, Reservation-based dynamic TDMA protocol for medical body area networks. IEICE Trans. Commun. 92(2), 387-395 (Feb.)

32. B. Yahya, J. Ben-othman, Towards a classification of energy aware MAC protocols for wireless sensor networks. Wirel. Commun. Mobile Comput. 9, 1572-1607 (Feb.)

33. O.N.C. Yilmaz, J. Hamalainen, S. Hamalainen, Self-optimization of random access channel in $3^{\text {rd }}$ generation partnership project long term evolution. Wiley Wirel. Commun. Mobile Comput. 11(12), 1507-1517 (Dec.)

34. S. Rahimian, M. Noori, M. Ardakani, An energy-efficient adaptive frameless ALOHA protocol. EURASIP J. Wirel. Commun. Netw. 1, 1-11 (Dec.)

35. A medium access protocol: ALOHA [Online]. https://www.slideshare.net/mobile/bhanutulya17/aloha-proto col-in-detail. Accessed 7 July 2019.

36. Chapter 3: random access networks [Online]. http://www.eeng.dcu.ie/ ee414/notes/ee414-09-slides-6.pdf

37. Difference between pure ALOHA and slotted ALOHA [Online]. https://techdifferences.com/difference-betwe en-pure-aloha-and-slotted-aloha.html. Accessed 16 July 2019.

38. M.B. Rasheed, N. Javaid, M. Imran, Z.A. Khan, U. Qasim, A. Vasilakos, Delay and energy consumption analysis of priority guaranteed MAC protocol for wireless body area networks. Wirel. Netw. 23(4), 1249-1266 (May )

39. D. K. Klair, K. W. Chin, and R. Raad, An investigation into the energy efficiency of pure and slotted aloha based REID anti-collision protocols. in Proc. IEEE 2007 International Symp. World of Wireless, Mobile and Multimedia Networks, Australia, Jun. 2007

40. L. Kleinrock, F. Tobagi, Packet switching in radio channels: Part 1-carrier sense multiple access modes and their throughput delay characteristics. IEEE Trans. Commun. 23, 1400-1416 (1975)

41. H. Harada, R. Prasad, Simulation and Software Radio for Mobile Communications (Norwood, Artech House, 2002), pp. 271-334

42. The variants of the CSMA protocols [Online]. http://www.mathcs.emory.edu/ cheung/Courses/455/Sylla bus/3a-MAC/csma2.html Accessed 21 July 2019.

43. M.Y. Darus, A. Kamarudin, N. Awang, and F.H. Ali.Analysis performance on contention-based MAC protocols in MANETs. in Proc. IEEE 2014 International Conf. World Congress on Information and Communication Technologies (WICT), 2014

44. Random Access Protocols - ALOHA, CSMA, CSMA/CA, and CSMA/ [Online]. https://www.studytonight.com/ post/random-access-protocols-aloha-csma-csmaca-and-csmacd. Accessed 24 November 2020.

45. CSMA/CA (Carrier sense multiple access/collision avoidance) [Online]. https://searchnetworking.techtarget .com/definition/CSMA-CA. Accessed 25 July 2019.

46. M. Younis and T. Nadeem, Energy efficient MAC protocols for wireless sensor networks. Technical Report, 2004.

47. T.T. Le, S. Moh, Hybrid multi-channel MAC protocol for WBANs with inter-WBAN interference mitigation. Sensors 18(5), $1373(2018)$

48. B. Liu, Z. Yan, and C. W. Chen, A hybrid context-aware MAC protocol for wireless body area networks. in Proc. IEEE 13th 2011 International Conf. E-Health Networking, Applications and Services, pp. 213-216

49. R. Wang, H. Wang, H. E. Roman, Y. Wang, and D. Xu, A cooperative medium access control protocol for mobile clusters in wireless body area networks. in Proc. IEEE 2013 1st International Symp. Future Information and Communication Technologies for Ubiquitous Healthcare (Ubi-HealthTech), pp. 1-4

50. G. Fang and E. Dutkiewicz, Body MAC energy efficient TDMA-based MAC protocol for wireless body area networks. in Proc 9th International Symposium Communications and Information Technology, 2009

51. H. Li, J. Tan, Heartbeat-driven medium access control for body sensor networks. IEEE Trans. Inf Technol. Biomed. 14(1), 44-51 (Sept)

52. N.F. Timmons and W.G. Scanlon, An adaptive energy efficient MAC protocol for the medical body area networks. in Proc.1st International Conf. Wireless communication VITAE, pp. 587-593, 2009.

53. H. Su, X. Zhang, Battery-dynamics driven TDMA MAC protocols for wireless body-area monitoring networks in healthcare applications. IEEE J. Sel. Areas Commun. 27(4), 424-434 (2009) 
54. Y. Zhang. and G. Dolman, A new priority-guaranteed MAC protocol for emerging body area networks. in Proc. 5th International Conf. Wireless and Mobile Communications (ICWMC), pp.140-145, 2009.

55. J. Zheng, M.J. Lee, A comprehensive performance study of IEEE 802154. Sensor Netw. Oper. 4, 218-237 (2006)

56. W. Ye, J. Heidemann, and D. Estrin. Medium access control with coordinated, adaptive sleeping for wireless sensor networks. IEEE/ACM Transactions on Networking, 2004. http://portal.acm.org/citation.cfm?id=10084 63.1008471 .

57. M. Chen, S. Gonzalez, A. Vasilakos, H. Cao, V.C. Leung, Body area networks: a survey. Mobile Netw. Appl. 16(2), 171-193 (Apr.)

58. R. Huang, Z. Nie, C. Duan, Y. Liu, L. Jia, and L. Wang, Analysis and comparison of the IEEE 802.15.4 and 802.15.6 wireless standards based on MAC layer. in Proc. International Conf. Health Information Science, May 2015, pp. 7-16, Springer, Cham.

59. IEEE Standard for Local and Metropolitan Area Networks Part 15:4: Low-Rate Wireless Personal Area Networks (LRWPANs), IEEE Standard Association, Piscataway, NJ, USA, 2011.

60. M.M. Alam, E.B. Hamida, Surveying wearable human assistive technology for life safety critical applications: standards, challenges and opportunities. Sensors 14, 9153-9209 (2014)

61. P. Park, P. Di Marco, C. Fischione, K.H. Jahansson, Modelling and optimization of the IEEE 802154 protocol for reliable and timely communications. IEEE Trans. Parallel Distrib. Syst. 24(3), 550-564 (2013)

62. L. Hughes, X. Wang, T. Chen, A review of protocol implementations and energy efficient cross-layer design for wireless body area networks. Sensors 12(11), 14730-14773 (Oct.)

63. M. Singh and A. K. Verma, Comparative analysis of IEEE 802.15. 4 and IEEE 802.15. 6 Standards. in Proc. IEEE 2014 International Conf. on Computational Intelligence and Communication Networks, Bhopal, India, Nov. 14, pp. 370-373

64. . Baradai, L.C. Fourati, and L. Kamou, Performance analysis of medium access control protocol for wireless body area networks. in Proc. IEEE 27th 2013 International Conf. Advanced Information Networking and Applications Workshops, Barcelona, Spain, Mar. 2013, pp. 916-921

65. F. Ullah, A.H. Abdullah, O. Kaiwartya, S. Kumar, MMd. Arshad, Medium access control (MAC) for wireless body area network (WBAN): superframe structure, multiple access technique, taxonomy and challenges. Hum. Cent. Comput. Inf. Sci. 34(7), 1-39 (2017)

66. M. Cicioglu, A. Calhan, HUBsFLOW: a novel interface protocol for SDN-enabled WBANs. Comput. Netw. 160, 105-117 (Sep.)

67. K.S. Kwak, S. Ullah, and N. Ullah, An overview of IEEE 802.15. 6 standard. in Proc. 3rd IEEE 2010 International Symp. Applied Sciences in Biomedical and Communication Technologies (ISABEL), Rome, Italy, Nov. 2010, pp. 1-6

68. S. Movassaghi, M. Abolhasan, J. Lipman, D. Smith, A. Jamalipour, Wireless body area networks: a survey. IEEE Commun. Surv. Tutorials 16(3), 1658-1686 (2014)

69. S. Ullah, M. Mohaisen, M.A. Alnuem, A review of IEEE 802.15.6 MAC, PHY, and security specifications. Int. J. Distrib. Sensor Netw. 9(4), 1-12 (2013)

70. R. Huang, Z. Nie, C. Duan, Y. Liu, L. Jia, and L. Wang, Analysis and comparison of the IEEE 802.15.4 and IEEE 802.15.6 wireless standards based on MAC layer. in Proc. Singer International Conf. Health Information Science, Switzerland, May 2015, pp. 7-16.

71. M. Cicioglu, A. Calhan, Performance analysis of IEEE 802.15.6 for underground disaster cases. Comput. Stand. Interfaces 66, 1-10 (2019)

72. F. Di Franco, Y. Ge, and I. Tinnirello, On-body and off-body body transmit power control in IEEE 802.15.6 schedules access networks. in Proc. IEEE 25th International Symp. Personal, Indoor, and Mobile Radio Communication, Washington, DC, USA, Sept. 2014, pp. 1245-1258.

73. Wireless LAN Medium Access Control (MAC) and Physical Layer (PHY) Specification, IEEE Standard Association, Piscataway, NJ, USA, 2012.

74. J.C. Haartsen, The Bluetooth Radio System. IEEE Personal Communications, pp. 28-36. Feb. 2000

75. 76Bluetooth [Online]. https://www.geeksforgeeks.org/bluetooth/. Accessed November 2020.

76. M. Kalia, D. Bansal, and R. Shorey, Data scheduling and SAR for Bluetooth MAC. in Proc. 51 st IEEE 2000 International Conf. Vehicular Technology (Cat. No. 00CH37026), Tokyo, Japan, May 2000, pp. 716-720.

77. M.U. Rehman, Y. Gao, Z. Wang, J. Zhang, Y. Alfadhi, X. Chen, C.G. Parini, Z. Ying, T. Bolin, Investigation of onbody Bluetooth transmission. IET Microw. Antennas Propag. 4(7), 871-880 (Jul.)

78. 3 Key Factors That Determine the Range of Bluetooth [Online]. http://www.bluetooth.com/blog/3-key-facto rs-that-determinethe-range-of-bluetooth/. Accessed April 162020

79. Y. Zatout, Using wireless technologies for healthcare monitoring at home: a survey. in Proc. 2012 IEEE 14th International Conf. e-Health Networking, Applications and Services (Healthcom), Beijing, China, Oct. 201210, pp. 383-386

80. 802.11 Working Group of the LAN/MAN Standards Committee of the IEEE Computer Society, IEEE P802.11 ah/ D10, IEEE, 2016.

81. The editors of IEEE 802.11, Wireless LAN Medium Access Control (MAC) and Physical Layer (PHY) Specification, 1997.

82. E.S. Jung and N. H. Vaidya, An energy efficient MAC protocol for wireless LANs. in Proceedings of the 21st Annual Joint Conf. IEEE Computer and Communications Societies Jun. 2002, pp. 1756-1764.

83. H. Zhai, Y. Kwon, and Y. Fang., Performance analysis of IEEE 802.11 MAC protocols in wireless LANs. Wirel. Commun. Mobile Comput. 4(8), 917-931, 2004

84. WiFi MAC protocol [Online]. http://webcache.googleusercontent.com/search?q=cache:wXHZ7rArpr MJ:www.cse.unt.edu/ rdantu/FALL_2018_WIRELESS_NETWORKS/WiFi_MAC.ppt $+\& \mathrm{~cd}=15 \& \mathrm{hl}=\mathrm{en} \& \mathrm{ct}=\mathrm{clnk} \&$ $\mathrm{gl}=\mathrm{za}$. Accessed July 302019. 
85. E. Ferro, F. Potorti, Bluetooth and Wi-Fi wireless protocols: a survey and a comparison. IEEE Wirel. Commun. 12(1), 12-26 (Mar.)

86. X. Lei, S.H. Rhee, Improving the IEEE 80211 power-saving mechanism in the presence of hidden terminals. EURASIP J. Wirel. Commun. Netw. 26, 1-10 (2016)

87. S.N. Marwat, Y. Mehmood, F. Ullah, A. Khan, S. Khan, S. Ahmed, D. Kwak, A. Nazir, A mobile Wi-Fi based scheduling of cyber-physical systems in healthcare. Electronics $\mathbf{9}(2), 247$ (Feb.)

88. Network Smart Body Area, Low Complexity Medium Access Control (MAC) for SmartBAN, ETSI TC SmartBAN TS 103325V1.1.1 2015, [Online]. http://www.etsi.org/deliver/etsi_ts/103300_,103399(103325). Accessed April 172020.

89. T. Paso, H. Tanaka, M. Hamalainen, W. H. Chin, R. Matsuo, and J. Haapola, An overview of ETSI TC SmartBAN MAC protocol. in Proc. IEEE2015 9th International Symp. Medical Information and Communication Technology, Mar. 2015, pp. 10-14.

90. R. Kufakunesu, G.P. Hancke, A.M. Abu-Mahfouz, A survey on adaptive data rate optimization in LoRaWAN: recent solutions and major challenges. Sensors MDPI 20, 1-25 (2020)

91. U. Raza, P. Kulkarni, M. Sooriyabandara, Low power wide area network: an overview. IEEE Commun. Surveys Tuts. 19(2), 855-873 (2017)

92. E. Morin, M. Maman, R. Guizzetti, A. Dauda, Comparison of the device lifetime in wireless networks for the internet of things. IEEE Access 5, 7097-7114 (Apr.)

93. C. Goursaud, J.M. Gorce, Dedicated networks for loT: PHY/MAC state of the art and challenges. EAI Endorsed Trans. Internet of Things 1, 1-12 (Oct.)

94. A. Sivasubramanian, S.P.K. Babu, A review of MAC scheduling algorithms in LTE system. Int. J. Adv. Sci. Eng. Inf. Technol. 7(3), 1056-1068 (2017)

95. M. Huang, A. Liu, T. Wang, A.V. Vasilakos, An effective servie-oriented networking management architecture for 5G-enabled internet of things. Comput. Netw. 18, 1-17 (Mar.)

96. A. Osseiran, J.F. Monserrat, F. Marsch, G Mobile and Wireless Communications Technology (Cambridge University Press, Cambridge, 2016).

97. A. Ali, W. Hamouda, M. Uysal, Next generation M2M cellular networks: challenges and practical Considerations. IEEE Commun. Mag. 53, 18-24 (2015)

98. E. M. Ang, K. K. Wee, Y. H. Pang, and K. K. Phang, A performance analysis on packet scheduling schemes based on an exponential rule for real-time traffic in LTE. EURASIP J. Wirel. Commun. Netw. 2015.

99. O. Liberg, M. Sundberg, E. Wang, J. Bergman, J. Sachs, Cellular Internet of Things: Technologies, Standards, and Performance (Elsevier Academic Press, Cambridge, 2017).

100. LTE overview [Online]. https://www.tutorialspoint.com/lte/lte_overview.htm. Accessed July 312019.

101. Medium access control in specification, LTE, MAC [Online]. http://4g5gworld.com/specification/mediu m-access-control-mac. Accessed July 312019.

102. Y. J. Zhang and S. C. Liew, Link-adaptive largest weighted-throughput packet scheduling for real-time traffics in wireless OFDM networks. in Proc. IEEE International Conf. Global Telecommunications, 2005.

103. R. Ratasuk, N. Mangalvedhe, Y. Zhang, M. Robert, and J. P. Koskinen, Overview of narrowband IoT in LTE Rel13. in Proc. IEEE 2016 International Conf. Standards for Communications and Networking (CSCN), Berlin, 2016, pp. $1-7$

104. A. D. Zayas and P. Merino, The 3GPP NB-IoT system architecture for the Internet of Things. in Proc. IEEE International Conf. Communications Workshops (ICC Workshops), Paris, France, May 2017, pp. 277-282.

105. The 3rd Generation Partnership Project-3GPP TS36-213. Evolved Universal Terrestrial Radio Access (EUTRA) and Evolved Universal Terrestrial Radio Access Network (EUTRAN); Physical Layer Procedures [Online]. https:// www.etsi.org/deliver/etsi_ts/136200_136299/136213/14.02.00_60/ts_136213v140200p.pdf. Accessed July 31 2019.

106. L. Zhang, Y.C. Liang, M. Xiao, Spectrum sharing for internet of things: a survey. IEEE Wirel. Commun. 26(3), $132-139$ (Dec.)

107. C.B. Mwakwata, H. Malik, M.M. Alam, Y.L. Moullec, S. Parand, S. Mumtaz, Narrowband internet of things (NBloT): from physical (PHY) and media access control (MAC) layers perspectives. Sensors 19, 2613 (2019)

108. C. Fehri, M. Kassab, S. Abdellatif, P. Berthou, and A. Belghith, LoRa technology: MAC layer operations and research issues. in Proc. 4th International Workshop on Recent Advances on Internet of Things (RAMCOM), Porto, Portugal, May 2018, pp. 1-8.

109. C.C. Enz, A.E. Hoiydi, J.D. Decotignie, V. Peiris, WiseNET: an ultralow power wireless sensor network solution. IEEE Comput. 37(8), 62-70 (Aug.)

110. P. Le-Huy and S. Roy, Low-power $2.4 \mathrm{GHz}$ wake-up radio for wireless sensor networks. in Proc. IEEE 2008 International Conf. Wireless and Mobile Computing Networking and Communications, Avigon, France, Oct. 2008. pp. 13-18

111. H. Al-Mishmish, A. Alkhayyat, H.A. Rahim, D.A. Hammood, R.B. Ahmad, Q.H. Abbasi, Critical data-based incremental cooperative communication for wireless body area network. Sensors 18, 3661 (Oct.)

112. S. Bhandari, S. Moh, A priority-based adaptive mac protocol for wireless body area networks. Sensors $\mathbf{1 6}(3)$ 401 (2016)

113. M. Shakir, O. Rehman, M. Rahim, N. Alrajeh, Z. Khan, M. Khan, I. Niaz, N. Javaid, Performance optimization of priority assisted csma/ca mechanism of 802.15.6 under saturation regime. Sensors 16(9), 1421 (2016)

114. M. Ndiaye, G.P. Hancke, A.M. Abu-Mahfouz, Software defined networking for improved wireless sensor network management: a survey. Sensors 17(5), 1031 (2017)

115. S. Bhandari, S. Moh, A survey of MAC protocols for cognitive radio body area networks. Sensors 15(4), 9189-9209 (Apr.)

116. H. Karl, and A. Willig, MAC Protocols. Protocols and Architectures for Wireless Sensor Networks. Wiley, West Sussex, pp. 111-148. 
117. M.R. Ahmad, E. Dutkiewicz, X. Huang, A survey of low duty cycle MAC protocols in wireless sensor networks. IntechOpen Emerg. Commun. Wirel. Sensor Netw. (2010). https://doi.org/10.5772/10512

118. R. Khan, M.M. Alam, T. Paso, J. Haapola, Throughput and Channel Aware MAC Scheduling for SmartBAN Standard. IEEE Access J. 7, 63133-63145 (2019)

119. J. Wang, Y. Xie, and Q. Yi, An all dynamic mac protocol for wireless body area network. in 11th International Conf. Wireless Communications, Networking and Mobile Computing (WiCOM 2015), Shanghai, China, Sep. 2015, pp. 1-6.

120. A. Alkhayyat, M.S. Mahmoud, Novel cooperative MAC aware network coding under log-normal shadowing channel model in wireless body area network. Int. J. Commun. Antenna Propag. 9(3), 198-206 (Jun.)

121. T.M. Chiwewe, G.P. Hancke, Fast convergence cooperative dynamic spectrum access for cognitive radio networks. IEEE Trans. Ind. Inform. 14(8), 3386-3394 (2018)

122. A.J. Onumanyi, A.M. Abu-Mahfouz, G.P. Hancke, Amplitude quantization method for autonomous threshold estimation in self-reconfigurable cognitive radio systems. Phys. Commun. 44, 1-18 (2020)

123. X. He and F. Y. Li, An optimal energy efficient cooperative retransmission MAC scheme in wireless networks. in Proc. 2nd International Conf. Wireless Commun. Veh. Technol. Inf. Theory Aerosp. Electron. Syst. Technl. (Wireless VITAE), Mar. 2011, pp. 1-5.

124. M. Al Ameen, N. Ullah, M.S. Chowdhury, S.M.R. Islam, K. Kwak, A power efficient MAC protocol for wireless body area networks. EURASIP J. Wirel. Commun. Netw. 33(1), 1-17 (2012)

125. T. Hayajneh, G. Almashagbeh, S. Ullah, A.V. Vasilakos, A survey of wireless technologies coexistence in WBAN: analysis and open research issues. Wireless Netw. 20(8), 2165-2199 (Nov.)

126. B. Mbarek, M. Ge, T. Pitner, An efficient mutual authentication scheme for internet of things. Internet of Things (Elsevier) 9, 1-9 (Jan.)

127. S. Challa, A.K. Das, P. Gope, N. Kumar, F. Wu, A.V. Vasilakos, Design and analysis of authenticated key agreement scheme in cloud-assisted cyber-physical systems. Fut. Gen. Comput. Syst. 108, 1267-1286 (Jul.)

128. M. Wazid, A.K. Das, V. Bhat, A.V. Vasilakos, LAM-CloT: Lightweight authentication mechanism in cloud-based IoT environment. J. Netw. Comput. Appl. 150, 1-23 (Jan.)

129. B. Mbarak, M. Ge, T. Pitner, An efficient mutual authentication scheme for internet of things. Internet Things. 9, $100160(2020)$

130. M. Ndiaye, S.S. Oyewobi, A.M. Abu-Mahfouz, G.P. Hancke, A.M. Kurien, K. Djouani, IoT in the wake of COVID-19: a survey on contributions, challenges and evolution. IEEE Access 8, 186821-186839 (Oct.)

\section{Publisher's Note}

Springer Nature remains neutral with regard to jurisdictional claims in published maps and institutional affliations.

\section{Submit your manuscript to a SpringerOpen ${ }^{\circ}$ journal and benefit from:}

- Convenient online submission

- Rigorous peer review

- Open access: articles freely available online

- High visibility within the field

Retaining the copyright to your article

Submit your next manuscript at $\boldsymbol{\Delta}$ springeropen.com 